\title{
Use of Dynamic Incidence Angle for Glide Slope Control of Autonomous Parafoils
}

\author{
Nathan Slegers* \\ Department of Mechanical and Aerospace Engineering \\ University of Alabama in Huntsville \\ Huntsville, Alabama \\ Eric Beyer ${ }^{\dagger}$ and Mark Costello ${ }^{\ddagger}$ \\ School of Aerospace Engineering \\ Georgia Institute of Technology \\ Atlanta, Georgia
}

\begin{abstract}
Strickly speaking, most autonomous parafoil and payload aircraft possess only lateral control, achieved by right and left parafoil brake deflection. An innovative new technique to achieve direct longitudinal control through dynamic incidence angle changes is reported. Addition of this extra control channel requires simple rigging changes and an additional servo actuator. The ability of dynamic incidence angle to alter the glide slope of a parafoil and payload aircraft is demonstrated through a flight test program with a micro parafoil system. Results from the flight test program are synthesized and integrated into a 6 degreeof-freedom simulation. The simulation model is subsequently used to assess the utility of glide slope control to improve autonomous flight control system performance. Through Monte Carlo simulation, impact point statistics with and without glide slope control indicate that dramatic improvements in impact point statistics are possible using direct glide slope control.
\end{abstract}

\section{Nomenclature}

$x, y, z=$ Inertial positions of the system mass center.

$\phi, \theta, \psi=$ Euler roll, pitch and yaw angles.

$u, v, w=$ Velocity components of mass center in a body reference frame.

$p, q, r=$ Angular velocity components in a body reference frame.

$\Gamma \quad=$ Canopy incidence angle.

$\vec{F}_{W} \quad=$ Weight vector in a body reference frame.

$\vec{F}_{S} \quad=$ Payload drag vector in a body reference frame.

$\vec{F}_{A}, \vec{M}_{A}=$ Aerodynamic force and moment vectors in a body reference frame.

$\vec{F}_{A M}, \vec{M}_{A M}=$ Apparent mass force and moment vectors in a body reference frame.

$I_{T} \quad=$ Inertia matrix of total system.

$C_{D S} \quad=$ Payload drag coefficient.

$\Delta x_{c}, \Delta y_{c}, \Delta z_{c}=$ Distance vector components from mass center to point $\mathrm{C}$ in a body reference frame.

$\Delta x_{p}, \Delta y_{p}, \Delta z_{p}=$ Distance vector components from mass $\mathrm{C}$ to $\mathrm{P}$ in a canopy reference frame.

\footnotetext{
* Assistant Professor, Member AIAA.

${ }^{\dagger}$ Graduate Research Assistant, Member AIAA.

‡ Sikorsky Associate Professor, Associate Fellow AIAA.
} 
$S_{\omega}^{B}, S_{\omega}^{C}=$ Cross product matrix of the angular velocity expressed in a body and canopy reference frame.

$S_{C G, P}^{B} \quad=$ Cross product matrix of the vector from the mass center to canopy center of pressure.

$S_{C G, M}^{B}=$ Cross product matrix of the vector from the mass center to apparent mass center.

$S_{C G, C}^{B} \quad=$ Cross product matrix of the vector from the mass center to canopy rotation point.

$S_{V_{A}}^{C} \quad=$ Cross product matrix of the parafoil aerodynamic velcoity.

$S_{P}, S_{S}=$ Reference area of the parafoil canopy and payload.

$\bar{c} \quad=$ Canopy main chord.

$b \quad=$ Canopy span.

$\bar{d} \quad=$ Brake characteristic length.

$\vec{V}_{A / I} \quad=$ Aerodynamic velocity vector including wind of the parafoil canopy in an inertial reference frame.

$V_{A}, V_{S}=$ Total aerodynamic speed of the parafoil canopy and payload.

\section{Introduction}

Parafoil and payload systems are unique flight vehicles well suited to perform autonomous airdrop missions. These air vehicles are compact before parafoil deployment, lightweight, fly at low speed, and impact the ground with low velocity. The predominant control mechanism for parafoils is left and right brake deflection. When a right brake control input is executed, the right back corner of the parafoil canopy is pulled down by changing the length of the appropriate suspension lines. Canopy changes created by brake deflection subsequently cause predictable changes in aerodynamic loads which is leveraged for control of the vehicle. For most parafoils, deployment of the right brake causes a significant drag rise and a small lift increase on the right side of the parafoil canopy combined with slight right tilt of the canopy. The overall effect causes the parafoil to skid turn to the right when a right parafoil brake is activated. ${ }^{1}$ Longitudinal control is more difficult to achieve. Ware and Hassell showed symmetric deflection of brakes to an angle of 45 degrees as pitch control did not effectively change the trim angle-of-attack; it did cause an increase in the lift and drag values at trim conditions, but the lift-drag ratio remained effectively unchanged. ${ }^{2}$ Symmetric brake deflection to an angle of 90 degrees caused large changes in trim angle-of-attack with the canopy stalling and the lift-drag was reduced to a value of about 0.5. Human sky divers also use weight shift for both longitudinal and lateral control. By shifting weight fore and aft, glide slope can be actively controlled and permits very accurate trajectory tracking, to include very accurate ground impact point control in the presence of relatively high atmospheric winds.

The bulk of current autonomous parafoil and payload aircraft employ right and left brake deflection for control which strictly speaking permits only lateral control. These aircraft typically do not have a direct means of longitudinal control. Hence, autonomous controllers for these air vehicles are greatly challenged to track three dimensional trajectories and impact a specific ground target point. The usual means to create some semblance of altitude control is through a weaving maneuver back and forth across a desired trajectory path to "dump" altitude as progress is made along the desired path. ${ }^{3-10}$ Near the intended ground impact location, current autonomous systems either spiral over the target or S-turn to the target. A key to the success for these algorithms is accurate descent rate estimation which is difficult to accomplish and prone to error.

The work reported here creates a glide slope control mechanism intended for use on autonomous parafoil and payload aircraft. Rather than using weight shift, glide slope control is physically achieved by changing the longitudinal rigging of the parafoil and payload combination dynamically in flight. The extra degree of freedom of control requires simple rigging changes and the addition of one additional servo actuator to the system. A detailed description of the basic mechanical design of the glide slope control mechanism is provided below. Traditional parafoil dynamic models treat the canopy orientation fixed with respect to the payload. ${ }^{11-14}$ These traditional models allow effects such as apparent mass to be easily incorporated. A new 6 degree-of-freedom model is created that include changing canopy orientation with respect to the payload and model apparent mass effects in a complete manner. 
When combined with traditional right and left brake control, glide slope control is an attractive feature for autonomous parafoil and payload aircraft since it allows the flight control laws to directly correct descent rate thus eliminating the need for descent rate estimation and the resulting error induced into the final delivery error. The ability of this system to change glide slope in flight is demonstrated with flight test results for an exemplar micro parafoil and payload system. The micro parafoil and payload system is fitted with a data logger equipped with a sensor suite that contains GPS, accelerometers, gyroscopes, barometric altimeter, magnetometers, and servo position so that the complete state of the payload along with all control inputs can be recorded. These flight test results are subsequently synthesized and incorporated into a 6 degree-of-freedom parafoil simulation and autonomous performance with and without glide slope control is reported. Monte Carlo simulations are performed to predict impact point statistics using only lateral control, and lateral/longitudinal control. Results indicate that a dramatic improvement in impact point statistics is realized with the addition of glide slope control.

\section{Parafoil Dynamic Model}

Figure 1 shows a schematic of a parafoil and payload system. With the exception of movable parafoil brakes, the parafoil canopy is considered to be a fixed shape once it has completely inflated. The combined system of the parafoil canopy and the payload are modeled with 6 DOF, including three inertial position components of the total system mass center as well as the three Euler orientation angles. Orientation of the parafoil canopy with respect to the payload is defined as the incidence angle $\Gamma$ and is considered a control variable. Rotation of the canopy about point $C$ allows tilting of the canopy lift and drag vectors resulting in changes in the equilibrium glide slope.

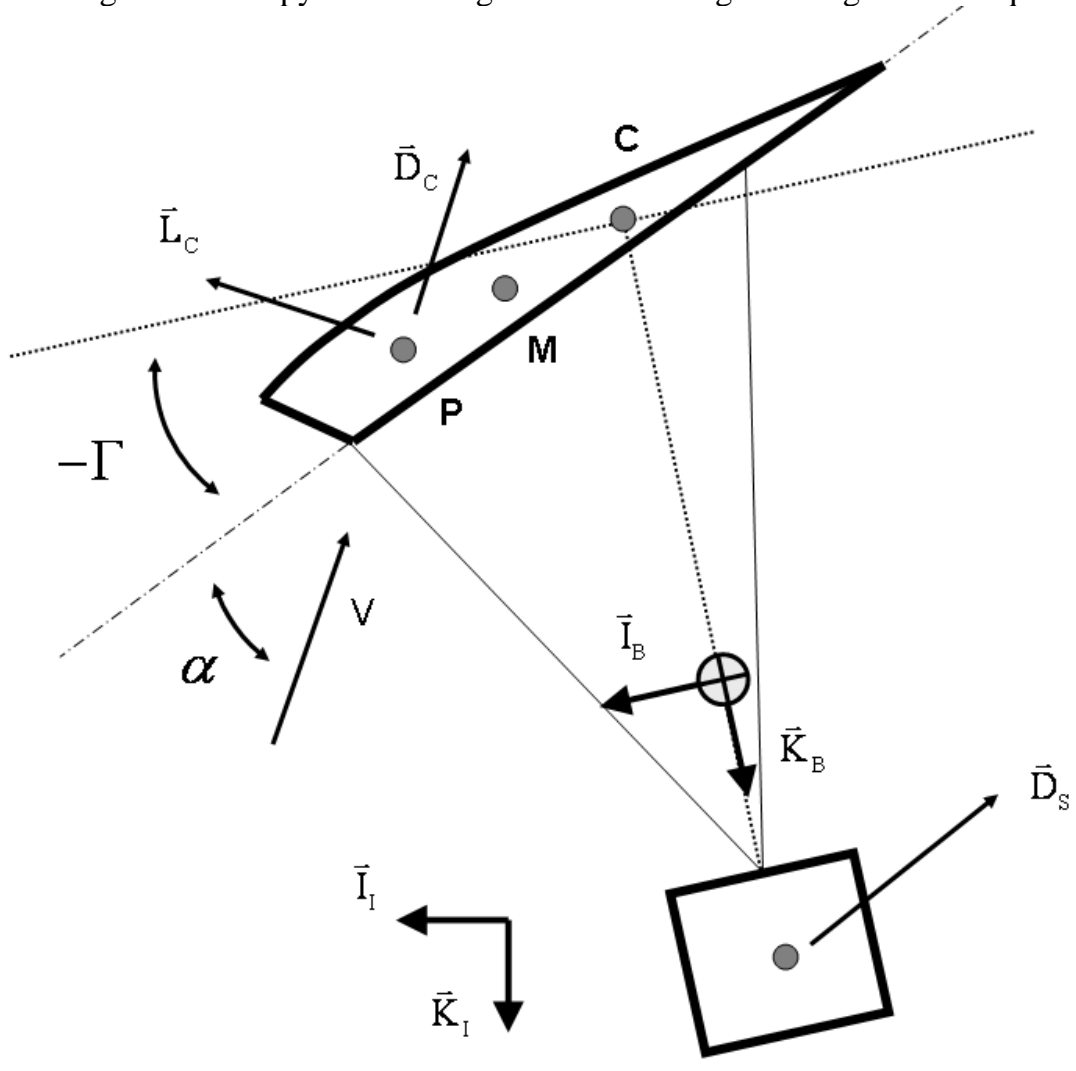

Figure 1. Parafoil and Payload Schematic

The kinematic equations for the parafoil and payload system are provided in Eqs. (1) and (2). The common shorthand notation for trigonometric functions is employed where $\sin (\alpha) \equiv s_{\alpha}, \cos (\alpha) \equiv s_{\alpha}$ and $\tan (\alpha) \equiv t_{\alpha}$. 


$$
\begin{gathered}
\left\{\begin{array}{c}
\dot{x} \\
\dot{y} \\
\dot{z}
\end{array}\right\}=\left[T_{I B}\right]^{T}\left\{\begin{array}{c}
u \\
v \\
w
\end{array}\right\} \\
\left\{\begin{array}{c}
\dot{\phi} \\
\dot{\theta} \\
\dot{\psi}
\end{array}\right\}=\left[\begin{array}{ccc}
1 & s_{\phi} t_{\theta} & c_{\phi} t_{\theta} \\
0 & c_{\phi} & -s_{\phi} \\
0 & s_{\phi} / c_{\theta} & c_{\phi} / c_{\theta}
\end{array}\right]\left\{\begin{array}{l}
p \\
q \\
r
\end{array}\right\}
\end{gathered}
$$

The matrix $T_{I B}$ represents the transformation matrix from an inertial reference frame to the body reference frame.

$$
T_{I B}=\left[\begin{array}{ccc}
c_{\theta} c_{\psi} & c_{\theta} S_{\psi} & -s_{\theta} \\
s_{\phi} s_{\theta} c_{\psi}-c_{\phi} s_{\psi} & s_{\phi} s_{\theta} s_{\psi}+c_{\phi} c_{\psi} & s_{\phi} c_{\theta} \\
c_{\phi} s_{\theta} c_{\psi}+s_{\phi} s_{\psi} & c_{\phi} s_{\theta} s_{\psi}-s_{\phi} c_{\psi} & c_{\phi} c_{\theta}
\end{array}\right]
$$

The dynamic equations are formed by summing forces and moments about the system CG both in the body reference frame and equating to the time derivative of linear and angular momentum respectively.

$$
\begin{gathered}
\left\{\begin{array}{c}
\dot{u} \\
\dot{v} \\
\dot{w}
\end{array}\right\}=\frac{1}{m}\left(\vec{F}_{W}+\vec{F}_{A}+\vec{F}_{S}+\vec{F}_{A M}\right)-S_{\omega}^{B}\left\{\begin{array}{l}
u \\
v \\
w
\end{array}\right\} \\
\left\{\begin{array}{c}
\dot{p} \\
\dot{q} \\
\dot{r}
\end{array}\right\}=\left[I_{T}\right]^{-1}\left(\vec{M}_{A}+\vec{M}_{A M}+S_{C G, P}^{B} \vec{F}_{A}+S_{C G, S}^{B} \vec{F}_{S}+S_{C G, M}^{B} \vec{F}_{A M}-S_{\omega}^{B}\left[I_{T}\right]\left\{\begin{array}{l}
p \\
q \\
r
\end{array}\right\}\right)
\end{gathered}
$$

The convention is used where the vector cross product of two vectors $\vec{r}=\left\{\begin{array}{lll}r_{x} & r_{y} & r_{z}\end{array}\right\}^{T}$ and $\vec{F}=\left\{\begin{array}{lll}F_{x} & F_{y} & F_{z}\end{array}\right\}^{T}$ both expressed in the A reference frame can be written as:

$$
S_{r}^{A} \vec{F}=\left[\begin{array}{ccc}
0 & -r_{z} & r_{y} \\
r_{z} & 0 & -r_{x} \\
-r_{y} & r_{x} & 0
\end{array}\right]\left\{\begin{array}{c}
F_{x} \\
F_{y} \\
F_{z}
\end{array}\right\}
$$

Forces appearing in Eq. (4) have contributions from weight, aerodynamic loads on the canopy and payload, and apparent mass. Weights contribution is given below in Eq. (7). 


$$
\vec{F}_{W}=m g\left\{\begin{array}{c}
-s_{\theta} \\
s_{\phi} c_{\theta} \\
c_{\phi} c_{\theta}
\end{array}\right\}
$$

Aerodynamic forces on the canopy appearing in Eq. (4) are expressed in the body reference frame; however they are a function of the aerodynamics velocities in the canopy frame. Defining $T_{B C}$ as the single axis transformation from the body to canopy reference frame by the incidence angle $\Gamma$ the aerodynamic velocity of the parafoil in the canopy frame is given in Eq. (8).

$$
\left\{\begin{array}{c}
\tilde{u} \\
\tilde{v} \\
\tilde{w}
\end{array}\right\}=\left[T_{B C}\right]\left(\left\{\begin{array}{c}
u \\
v \\
w
\end{array}\right\}+S_{\omega}^{B}\left(\left\{\begin{array}{l}
\Delta x_{c} \\
\Delta y_{c} \\
\Delta z_{c}
\end{array}\right\}+\left[T_{B C}\right]^{T}\left\{\begin{array}{l}
\Delta x_{p} \\
\Delta y_{p} \\
\Delta z_{p}
\end{array}\right\}\right)-\left[T_{I B}\right] \vec{V}_{A / I}\right)
$$

The aerodynamic angles then become $\alpha=\operatorname{atan}(\tilde{w} / \tilde{u})$ and $\beta=\operatorname{asin}\left(\tilde{v} / V_{A}\right)$. Equation (9) defines the canopy aerodynamic forces in the body reference frame using $T_{A C}$ as the transformation from aerodynamic to canopy frames by the angle $\alpha$. Payload drag is defined in a similar manner in Eq. (10), where $u_{S A}, v_{S A}$ and $w_{S A}$ are payload aerodynamic velocities in the body frame.

$$
\begin{gathered}
\vec{F}_{A}=\frac{1}{2} \rho V_{A}^{2} S_{P}\left[T_{B C}\right]^{T}\left[T_{A C}\right]\left\{\begin{array}{c}
C_{D 0}+C_{D \alpha 2} \alpha^{2} \\
C_{Y \beta} \beta \\
C_{L 0}+C_{L \alpha} \alpha+C_{L \alpha 3} \alpha^{3}
\end{array}\right\} \\
\vec{F}_{S}=-\frac{1}{2} \rho V_{S}^{2} S_{S} C_{D S}\left\{\begin{array}{c}
u_{S A} \\
v_{S A} \\
w_{S A}
\end{array}\right\}
\end{gathered}
$$

Moments appearing in Eq. (5) have contributions from aerodynamic moments, apparent inertia, and from forces on the canopy and payload. Aerodynamic moments expressed in the body frame are given in Eq. (11).

$$
\vec{M}_{A}=\frac{1}{2} \rho V_{A}^{2} S_{P}\left[T_{B C}\right]^{T}\left\{\begin{array}{c}
b\left(C_{l \beta} \beta+\left(b / 2 V_{A}\right) C_{l p} \tilde{p}+\left(b / 2 V_{A}\right) C_{l r} \tilde{r}\right)+C_{l \delta a}\left(\delta_{a} / \bar{d}\right) \\
\bar{c}\left(C_{m 0}+\left(\bar{c} / 2 V_{A}\right) C_{m q} \tilde{q}\right) \\
b\left(C_{n \beta} \beta+\left(b / 2 V_{A}\right) C_{n p} \tilde{p}+\left(b / 2 V_{A}\right) C_{n r} \tilde{r}\right)+C_{n \delta a}\left(\delta_{a} / \bar{d}\right)
\end{array}\right\}
$$

A body moving in a fluid puts the fluid in motion. The result from accelerating the fluid is a rate of change in both its linear and angular momentum. Typical aircraft having large mass to volume ratios have negligible effects from the mass of the accelerating fluid. Parafoils with small mass to volume ratios can experience large forces and moments from accelerating fluid called "apparent mass" and "apparent inertia" because they appear as additional mass and inertia values in the final equations of motion. Kinetic energy of the fluid can be written as 


$$
2 \mathrm{~T}=\mathrm{A} \tilde{u}^{2}+\mathrm{B} \tilde{v}^{2}+\mathrm{C} \tilde{w}^{2}+\mathrm{P} \tilde{p}^{2}+\mathrm{Q} \tilde{q}^{2}+\mathrm{R} \tilde{r}^{2}+2 \mathrm{~F}(\tilde{v} \tilde{r}+\tilde{w} \tilde{q})
$$

where it is assumed the canopy has two planes of symmetry, $x-z$ and $y-z$. Asymmetry about the $x-y$ plane is allowed to account for spanwise camber and the seven constants are defined by Lamb. ${ }^{15} \mathrm{~A}$ canopy of general shape may have has many as 21 constants defining the kinetic energy however; typical canopies will have two planes of symmetry reducing to only seven constants. Often spanwise camber is neglected and the canopy is approximated as an ellipsoid so that F becomes zero. The constant in Eq. (12) can be calculated numerically for a known shape or can be approximated as discussed in Refs. 15, 16 and 17. Forces and moments from apparent mass and inertia are found by relating the fluids momentum to its kinetic energy in a similar way as Lissman and Brown and are summarized in Eqs. (13) to (17). ${ }^{16}$

$$
\begin{gathered}
\vec{F}_{A M}=-\left[T_{B C}\right]^{T}\left(\left[I_{A M}\right]\left\{\begin{array}{c}
\dot{\tilde{u}} \\
\dot{\tilde{v}} \\
\dot{\tilde{w}}
\end{array}\right\}+\left[I_{F}\right]\left\{\begin{array}{c}
\dot{\tilde{p}} \\
\dot{\tilde{q}} \\
\dot{\tilde{r}}
\end{array}\right\}+S_{\omega}^{C}\left[I_{A M}\right]\left\{\begin{array}{c}
\tilde{u} \\
\tilde{v} \\
\tilde{w}
\end{array}\right\}+S_{\omega}^{C}\left[I_{F}\right]\left\{\begin{array}{c}
\tilde{p} \\
\tilde{q} \\
\tilde{r}
\end{array}\right\}\right) \\
\vec{M}_{A M}=-\left[T_{B C}\right]^{T}\left(\left[I_{F}\right]\left\{\begin{array}{c}
\dot{\tilde{u}} \\
\dot{\tilde{v}} \\
\dot{\tilde{w}}
\end{array}\right\}+\left[I_{A I}\right]\left\{\begin{array}{c}
\dot{\tilde{p}} \\
\dot{\tilde{q}} \\
\dot{\tilde{r}}
\end{array}\right\}+S_{\omega}^{C}\left[I_{F}\right]\left\{\begin{array}{c}
\tilde{u} \\
\tilde{v} \\
\tilde{w}
\end{array}\right\}+S_{\omega}^{C}\left[I_{A I}\right]+S_{V_{A}}^{C}\left[I_{F}\right]\left\{\begin{array}{c}
\tilde{p} \\
\tilde{q} \\
\tilde{r}
\end{array}\right\}\right)
\end{gathered}
$$

$$
\begin{aligned}
& {\left[I_{A M}\right]=\left[\begin{array}{lll}
\mathrm{A} & 0 & 0 \\
0 & \mathrm{~B} & 0 \\
0 & 0 & \mathrm{C}
\end{array}\right]} \\
& {\left[I_{A I}\right]=\left[\begin{array}{lll}
\mathrm{P} & 0 & 0 \\
0 & \mathrm{Q} & 0 \\
0 & 0 & \mathrm{R}
\end{array}\right]} \\
& {\left[I_{F}\right]=\left[\begin{array}{lll}
0 & 0 & 0 \\
0 & 0 & \mathrm{~F} \\
0 & \mathrm{~F} & 0
\end{array}\right]}
\end{aligned}
$$

Notice the forces and moments from apparent mass are a function of the canopy incidence angle. Equations (14) and (15) couple the linear and rotational dynamic in Eqs. (4) and (5). Final dynamic equations of motion are found substituting all forces and moments into Eqs. (4) and (5) resulting in the matrix solution in Eq. (18) to (20). The common convention is used for tensors of second rank such that $\left[I_{X}^{\prime}\right]=\left[T_{B C}\right]^{T}\left[I_{X}\right]\left[T_{B C}\right]^{T}$ for the quantities in Eqs. (15) to (17). 


$$
\begin{aligned}
& {\left[\begin{array}{c}
m \mathrm{I}+\left[I_{A M}^{\prime}\right] \\
\left.I_{T}^{\prime}+\left[I_{A I}^{\prime}\right]-\left[I_{F M}^{\prime}\right] S_{C G, M}^{B}\right] S_{C G, M}^{B} \\
S_{C G, M}^{B}\left[I_{A M}^{\prime}\right]+\left[I_{F}^{\prime}\right] \\
+S_{C G, M}^{B}\left(\left[I_{F}^{\prime}\right]-\left[I_{A M}^{\prime}\right]\right) S_{C G, M}^{B}
\end{array}\right]\left\{\begin{array}{c}
\dot{u} \\
\dot{v} \\
\dot{w} \\
\cdots \\
\dot{p} \\
\dot{q} \\
\dot{r}
\end{array}\right\}=\left\{\begin{array}{c}
\Phi \\
\cdots \\
\mathrm{M}
\end{array}\right\}} \\
& \Phi=\vec{F}_{A}+\vec{F}_{s}+\vec{F}_{W}-m S_{\omega}^{B}\left\{\begin{array}{l}
u \\
v \\
w
\end{array}\right\}-\left[T_{B C}\right]^{T} S_{\omega}^{C}\left(\left[I_{A M}\right]\left\{\begin{array}{c}
\tilde{u} \\
\tilde{v} \\
\tilde{w}
\end{array}\right\}+\left[I_{F}\right]\left\{\begin{array}{c}
\tilde{p} \\
\tilde{q} \\
\tilde{r}
\end{array}\right\}\right)-\left[I_{A M}^{\prime}\right] S_{\omega}^{B}\left[T_{I B}\right] \vec{V}_{A / I} \\
& \mathrm{M}=\vec{M}_{A}+S_{C G, P}^{B} \vec{F}_{A}+S_{C G, S}^{B} \vec{F}_{S}-S_{\omega}^{B}\left[I_{T}\right]\left\{\begin{array}{l}
p \\
q \\
r
\end{array}\right\}-S_{C G, M}^{B}\left[T_{B C}\right]^{T} S_{\omega}^{C}\left(\left[I_{A M}\right]\left\{\begin{array}{c}
\tilde{u} \\
\tilde{v} \\
\tilde{w}
\end{array}\right\}+\left[I_{F}\right]\left\{\begin{array}{c}
\tilde{p} \\
\tilde{q} \\
\tilde{r}
\end{array}\right\}\right) \\
& -\left[T_{B C}\right]^{T} S_{\omega}^{C}\left[I_{F}\right]\left\{\begin{array}{c}
\tilde{u} \\
\tilde{v} \\
\tilde{w}
\end{array}\right\}+\left[T_{B C}\right]^{T}\left(S_{\omega}^{C}\left[I_{A I}\right]+S_{V_{A / I}}^{C}\left[I_{F}\right]\right)\left\{\begin{array}{l}
\tilde{p} \\
\tilde{q} \\
\tilde{r}
\end{array}\right\}-\left(S_{C G, M}^{B}\left[I_{A M}^{\prime}\right]+\left[I_{F}^{\prime}\right]\right) S_{\omega}^{B}\left[T_{I B}\right] \vec{V}_{A / I}
\end{aligned}
$$

The matrix in Eq. (18) appears as an inertia matrix and satisfies many properties of a typical inertia matrix such as symmetry. In the case where all apparent mass and inertia effects are negligible Eq. (18) reduces to a block diagonal system where linear and rotational dynamic equations decouple. The effective apparent mass and inertia matrices $I_{A M}^{\prime}, I_{F}^{\prime}$ and $I_{A I}^{\prime}$ are functions of the canopy incidence angle so that dynamically changing the incidence angle for glide slope control results in varying apparent mass and inertia matrices. This is in contrast to conventional models where apparent mass and inertia coefficients are assumed constant.

\section{Test System}

The parafoil system tested is shown in Figs. 2 and 3 with the canopy deployed and undeployed respectively. The payload of the system consists of a 6 in $\times 6$ in $\times 18$ in cardboard box with two avionic boxes on either end. The upper avionics box, shown in Fig. 4a, consists of a data logger with battery, canopy pack, and a Hitec HS-311 servo used to release the packed parafoil. The upper box was designed to allow the top flaps of the cardboard box to flare out at a 45 deg angle to allow the undeployed system to be cone stabilized (see Fig. 3). The lower avionics box contains three Hitec HS-785 HB sail winches, a Hitec Electron 6 FM receiver, and a battery pack as shown in Fig. 4b. Sail winch 1 and 2 control the right and left brake lines, while sail winch 3 controls the front lines of the parafoil. The sail winch signals are mixed together so that as the front lines are pulled in, the brake lines are let out and vise versa. This allows the geometry of the canopy to accommodate for different incidence angles. Note the rear lines of the canopy remain fixed to the upper box while the brake lines and front lines run through the upper avionics box, to the sail winches in the lower avionics box. 


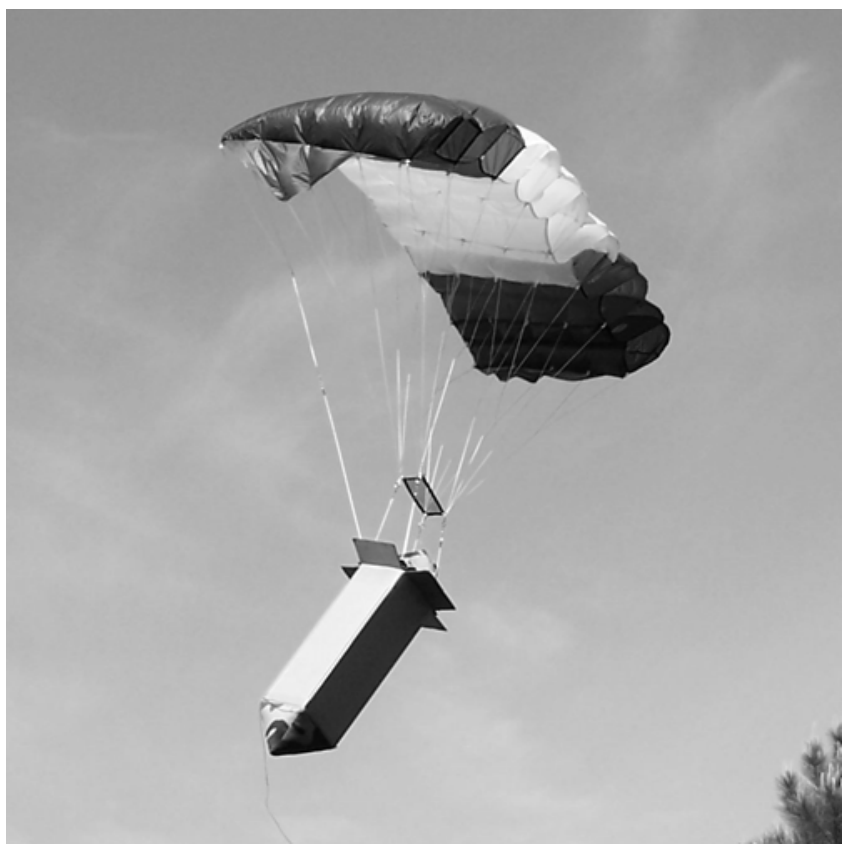

Figure 2. Test System with Deployed Parafoil

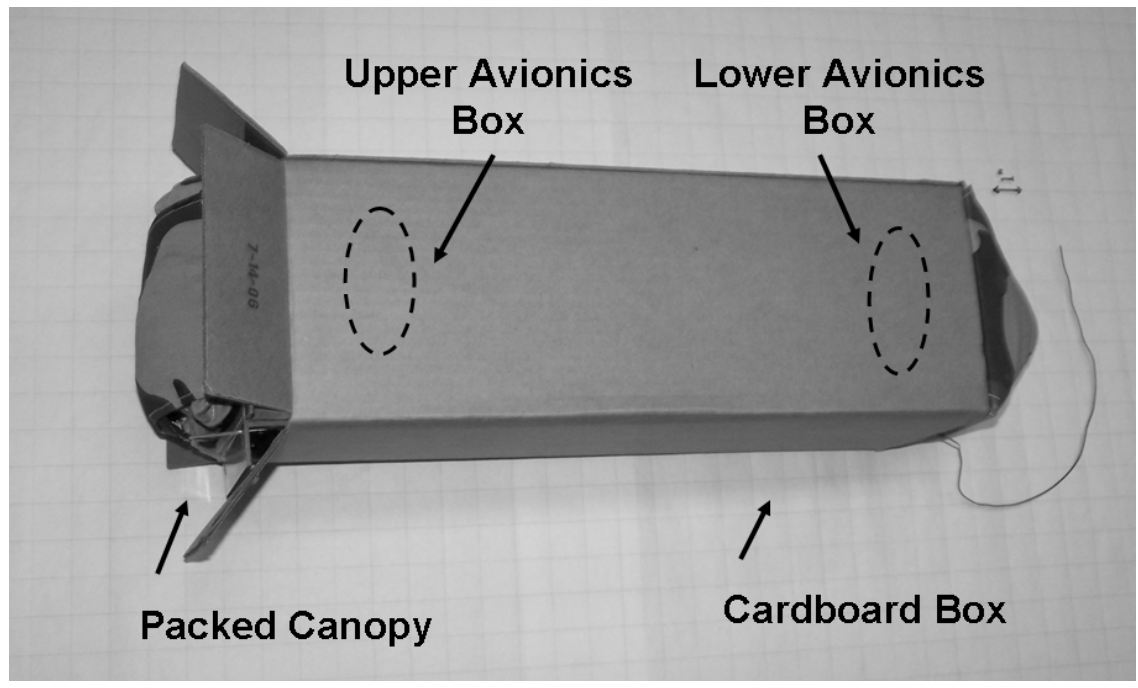

Figure 3. Test System with Undeployed Parafoil 

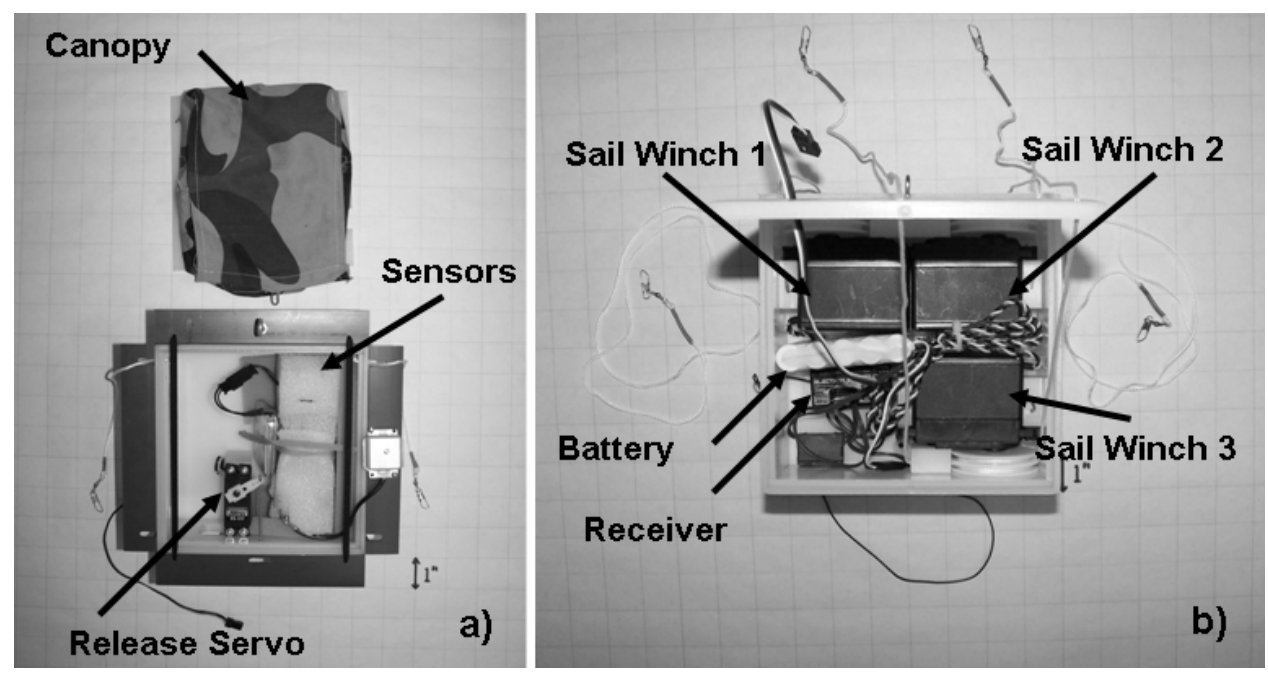

Figure 4. a) Upper Avionics Box b) Lower Avionics Box

As mentioned above; the incidence angle of the parafoil is changed using the three sail winches in the lower avionics box. An example of this incidence change is shown in Fig. 5. Two different parafoil systems were used in this study. Systems 1 and 2 differ mainly in their canopy thickness, leading edge geometry and payload weight. The dimensions of the systems are shown in Fig. 6 and outlined in Table 1.

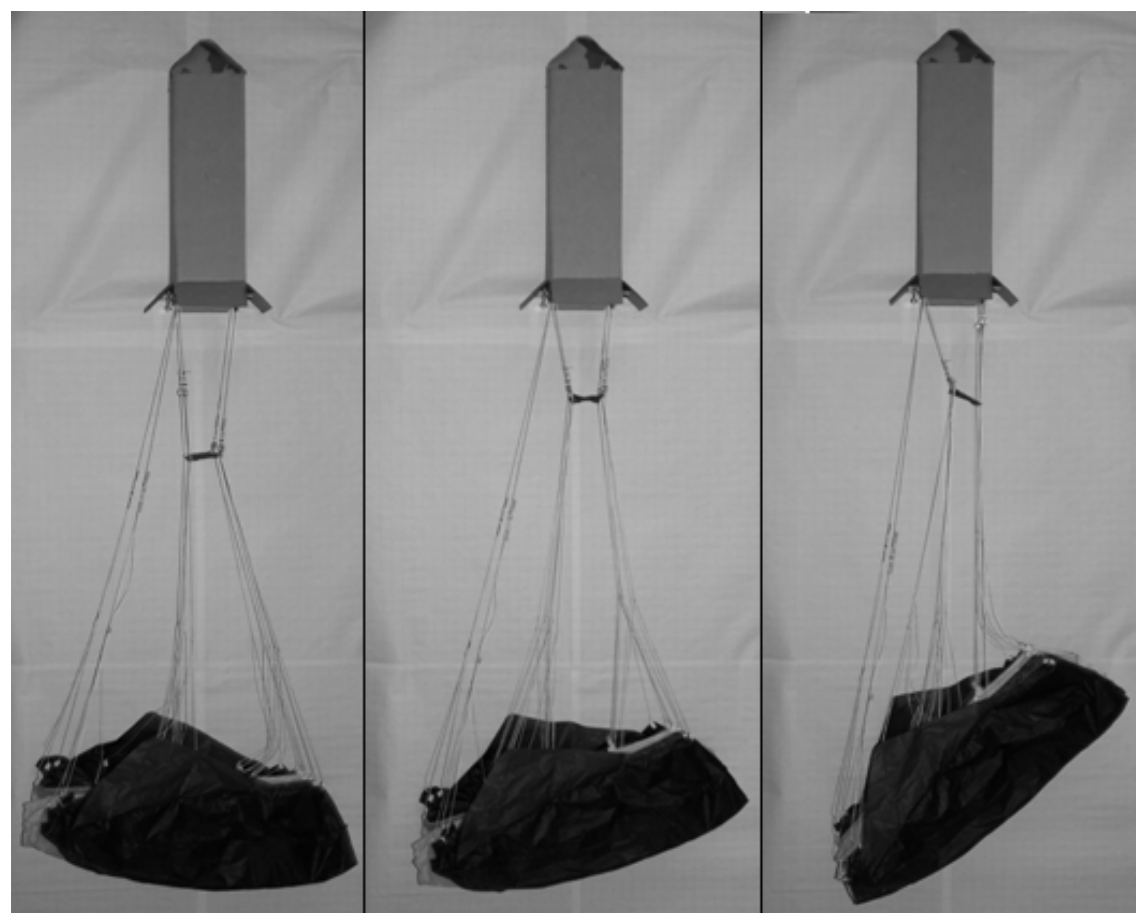

Figure 5. Parafoil Incidence Angle Change 

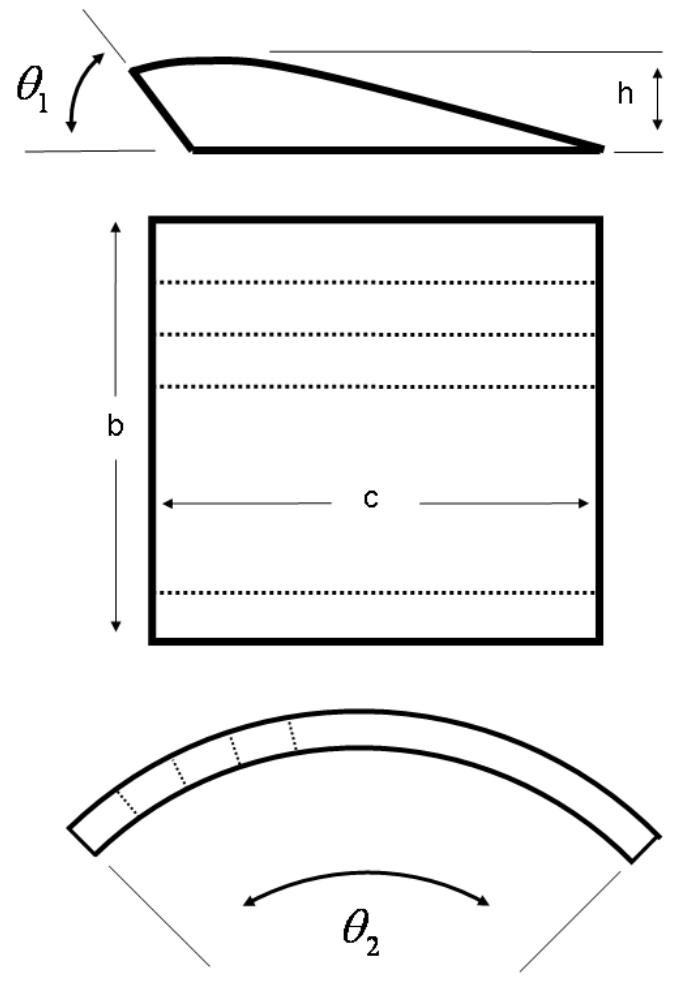

Figure 6. Canopy Geometry

Table 1. System Characteristics

\begin{tabular}{|c|c|c|}
\hline & System 1 & System 2 \\
\hline \hline$\theta_{I}(\mathrm{deg})$ & 80 & 50 \\
\hline$\theta_{2}(\mathrm{deg})$ & 45 & 45 \\
\hline $\mathrm{h}(\mathrm{ft})$ & 0.35 & 0.17 \\
\hline $\mathrm{b}(\mathrm{ft})$ & 4.5 & 5.0 \\
\hline $\mathrm{c}(\mathrm{ft})$ & 2.1 & 1.3 \\
\hline Weight(lbf) & 5.23 & 1.59 \\
\hline
\end{tabular}

\section{Flight Results}

Four flight tests were conducted, two for each system. System 1 was configured with a nominal incidence $\Gamma_{l}$ of 6 deg and once equilibrium was achieved the incidence angle was changed. In the first flight of system 1 the canopy was rotated down to an incidence $\Gamma_{2}$ of -24 deg, while during the second flight the canopy was rotated up to an incidence $\Gamma_{3}$ of $10 \mathrm{deg}$. System 2 was configured with a nominal incidence $\Gamma_{4}$ of -24 deg and was subsequently rotated down to an incidence $\Gamma_{5}$ of -44 deg for both the third and fourth flight tests. Results are shown after canopy opening in Figs. 7 to 9. Figure 7 shows results for the flight path where both altitude and distance have been nondimensionalized with respect to the initial altitude. The flight path angle $\lambda$ can be seen by the slope of the flight path in Fig. 7 and the glide slope (GS) is defined as $-1 / \tan (\lambda)$. Table 2 summarizes the four flight tests. 


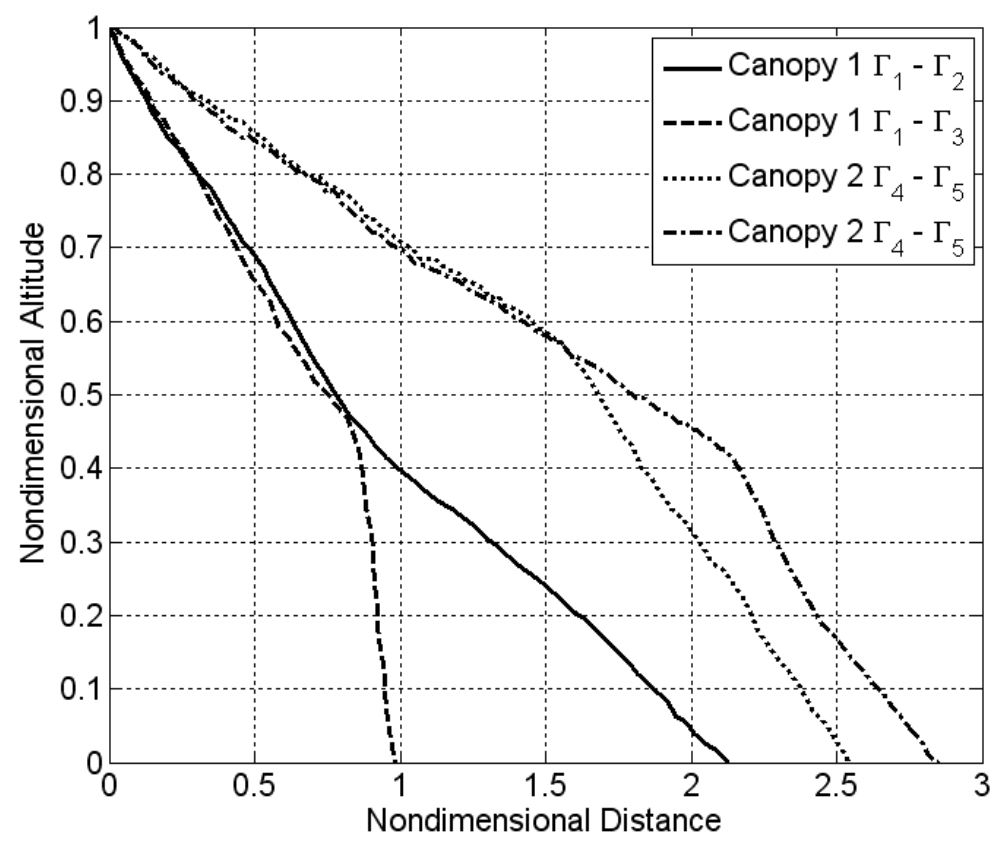

Figure 7. Flight Path Angle

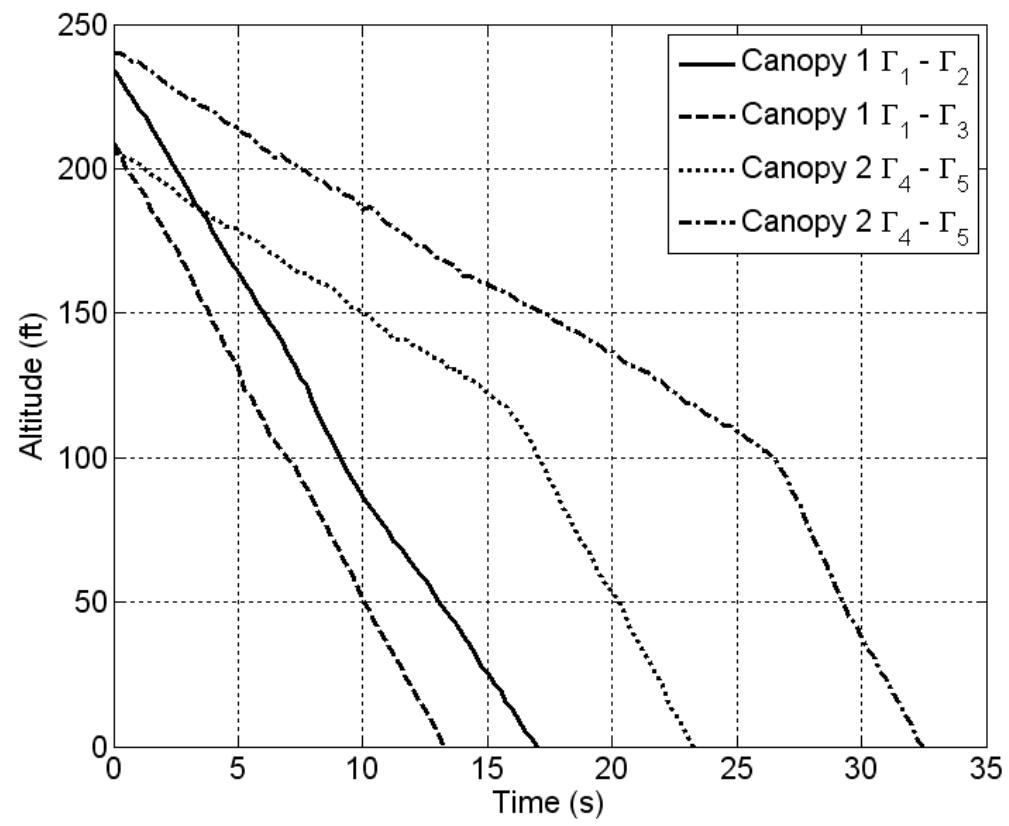

Figure 8. Altitude 


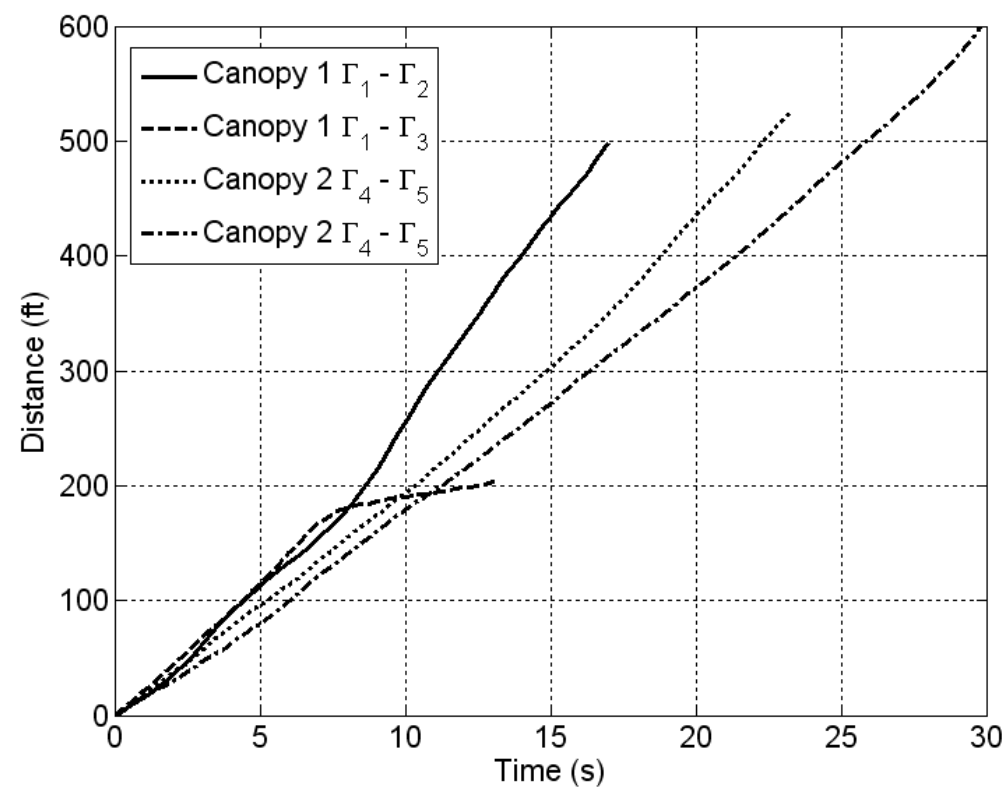

Figure 9. Distance Traveled

Table 2- Flight Test Summary

\begin{tabular}{|c|c|c|c|c|c|}
\hline & $\begin{array}{c}\text { System 1 } \\
\Gamma_{1}=-6 \mathrm{deg}\end{array}$ & $\begin{array}{c}\text { System } 1 \\
\Gamma_{2}=-24 \mathrm{deg}\end{array}$ & $\begin{array}{c}\text { System } 1 \\
\Gamma_{3}=10 \mathrm{deg}\end{array}$ & $\begin{array}{c}\text { System } 1 \\
\Gamma_{4}=-24 \mathrm{deg}\end{array}$ & $\begin{array}{c}\text { System 2 } \\
\Gamma_{5}=-44 \mathrm{deg}\end{array}$ \\
\hline \hline$\alpha(\mathrm{deg})$ & 28 & 6 & 70 & 10 & 5 \\
\hline Glide Slope & 1.45 & 2.46 & 0.28 & 3.70 & 1.94 \\
\hline Speed (ft/s) & 26 & 35 & 16 & 21 & 26 \\
\hline $\mathrm{C}_{\mathrm{L}}$ & 0.56 & 0.35 & 0.49 & 0.45 & 0.27 \\
\hline
\end{tabular}

Canopy 1 responds to a decrease in incidence from -6 to -24 deg with a 70 percent increases in GS, from 1.45 to 2.46. Increasing the incidence from -6 to 10 deg results in a stalled condition were the GS is decreased 89 percent. System 2 responds in an opposite manner with a decrease in incidence from -24 to -44 deg resulting in a 35 percent decrease in GS from 2.6 to 1.7. Differences are also observed in the vertical and forward velocity trends where for system 1 changing the incidence results in large forward speed changes with vertical speed remaining nearly unchanged while for system 2 the opposite is true. Results from system 1 were used to estimate $C_{L}$ and $C_{D}$ curves for the combined system defined by $C_{L 0}, C_{L \alpha}, C_{L \alpha 3}, C_{D 0}$, and $C_{D \alpha 2}$ as $0.28,0.68,-0.35,0.135$, and 0.95 respectively. The estimated values are consistent with results from Ware and Hassell who observed maximum lift to drag ratios near 2.5, high profile drag and low maximum lift coefficients when compared to a standard rigid wing. Figure 10 shows the estimated curves compared with the measured results for system 1 . Simulations of the estimated system GS are shown in Fig. 11 and are consistent with test data. 


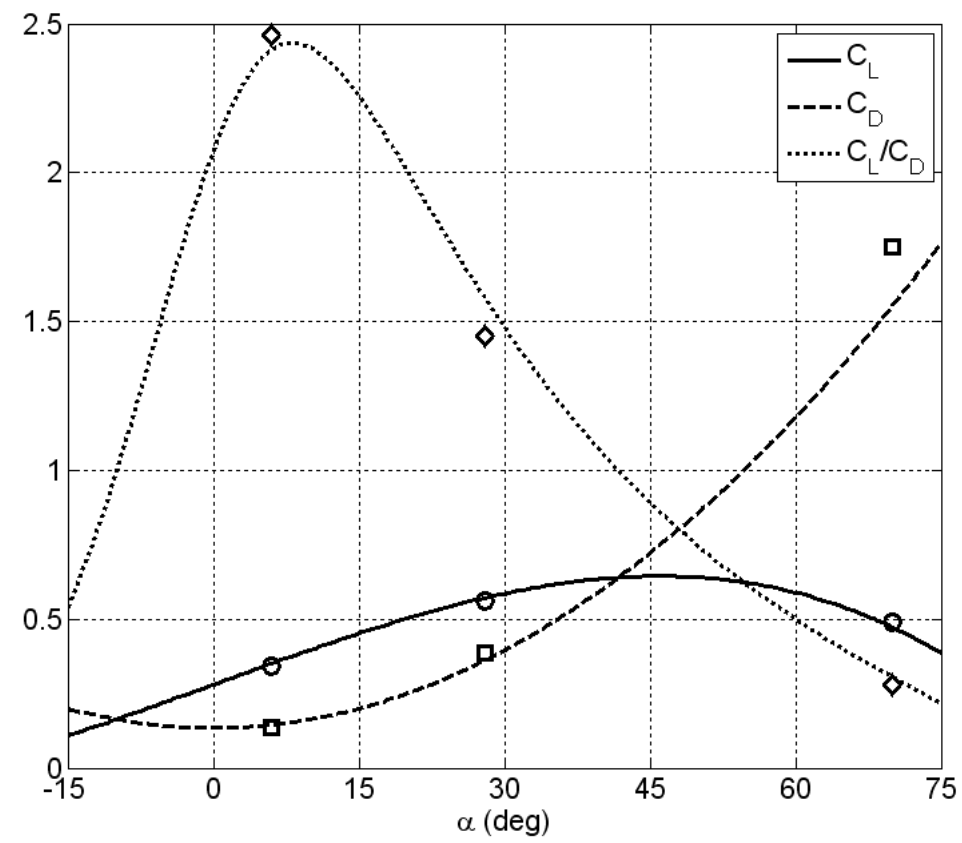

Figure 10. Lift Coefficient, Drag Coefficient, and $C_{L} / C_{D}$

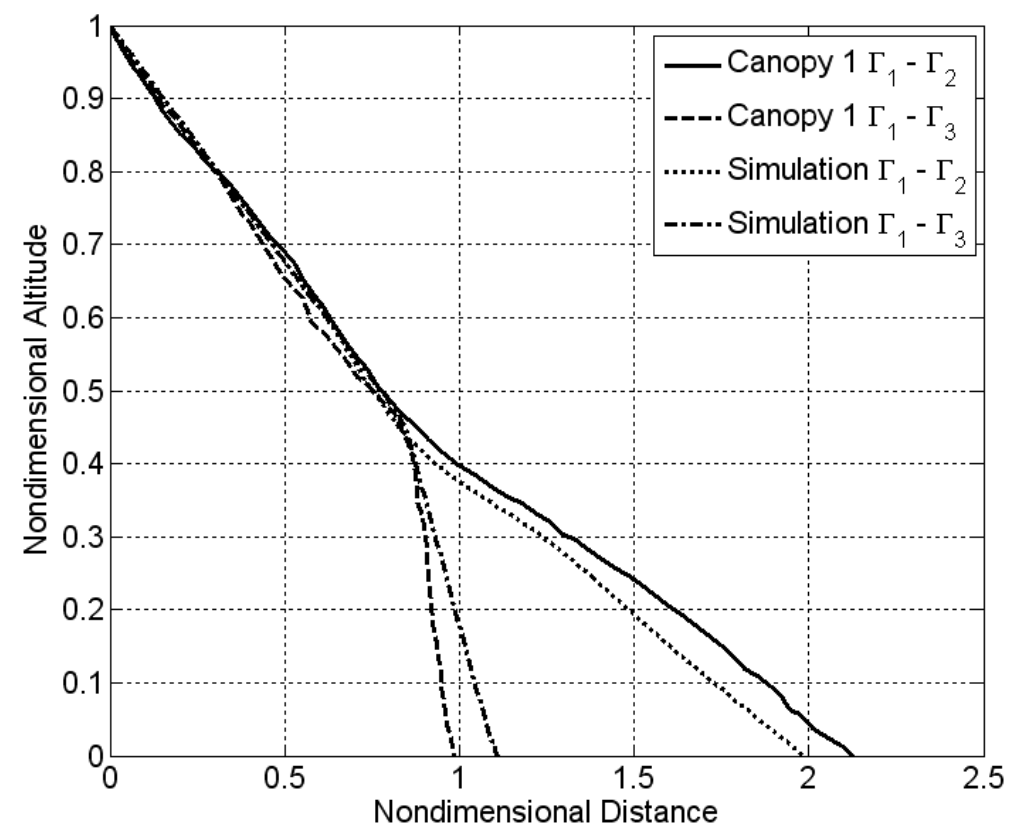

Figure 11. Comparison of Simulated and Measured GS for System 1

From Fig. 10 system 1 operates to the right of its maximum lift to drag ratio for all incidence angles resulting in an increased GS as the incidence is decreased. Decreasing the incidence of system 2 resulted in a decreased GS demonstrating system 2 operates to the left of its maximum lift to drag ratio. Noting that the maximum L/D occurs well before stall, its angle of attack can be estimated using Eq. (21). 


$$
\alpha_{L / D M A X}=\sqrt{\left(\frac{C_{L 0}}{C_{L \alpha}}\right)^{2}+\frac{C_{D 0}}{C_{D \alpha 2}}}-\frac{C_{L 0}}{C_{L \alpha}}
$$

Equation 21 shows the maximum $\mathrm{L} / \mathrm{D}$ angle of attack increases as $C_{L \alpha}$ increases and $C_{D \alpha 2}$ decreases, both occurring as the lifting surface efficiency factor increases. This is consistent with system 2 having a small thickness to chord ratio, larger aspect ratio and more rounded nose. Parafoils of higher efficiency will be able to operate to the left of their maximum L/D, while an inefficient canopy may operate to the right. It is demonstrated by systems 1 and 2 that a parafoil system can effectively operate on either side of the maximum $\mathrm{L} / \mathrm{D}$ angle of attack and have effective glide slope control. A system however may be arranged such that it operates near its maximum L/D, in such a case minimal glide slope control will result from the small slope of the L/D curve in this vicinity.

Simulations were completed for system 1 where the incidence was decreased from -6 to $-12,-18$ and -24 deg at 15 second intervals with each changes occurring linearly over a second. Figure 12 shows the glide slope dynamics persist for 5 seconds after each change in incidence. The GS initially decreases in response to the decreased lift from decreasing angle of attack before it increases as the speed increases. Changes in angle of attack and velocities are shown in Figs. 13 and 14, respectively. As incidence is decreased the angle of attack decreases approaching the maximum L/D where GS control authority diminishes. A nearly linear GS control mechanism can be implemented for system 1 by designing the nominal incidence to be -12 deg so that \pm 25 percent changes in GS can be achieved over a -6 to -18 incidence range. If maximum $\mathrm{L} / \mathrm{D}$ flight is desired system 1 can be flown at an incidence of -18 deg, however, the GS can only be effectively decreased.

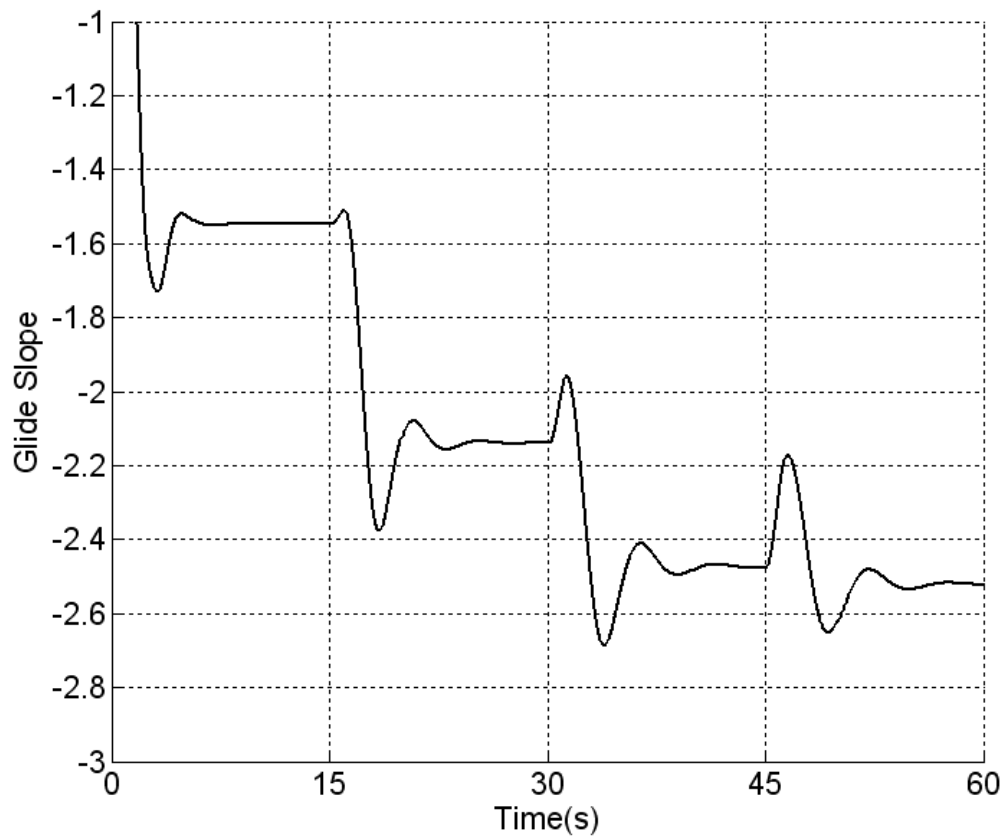

Figure 12. Simulated GS Varying Incidence $\Gamma$ of System 1 


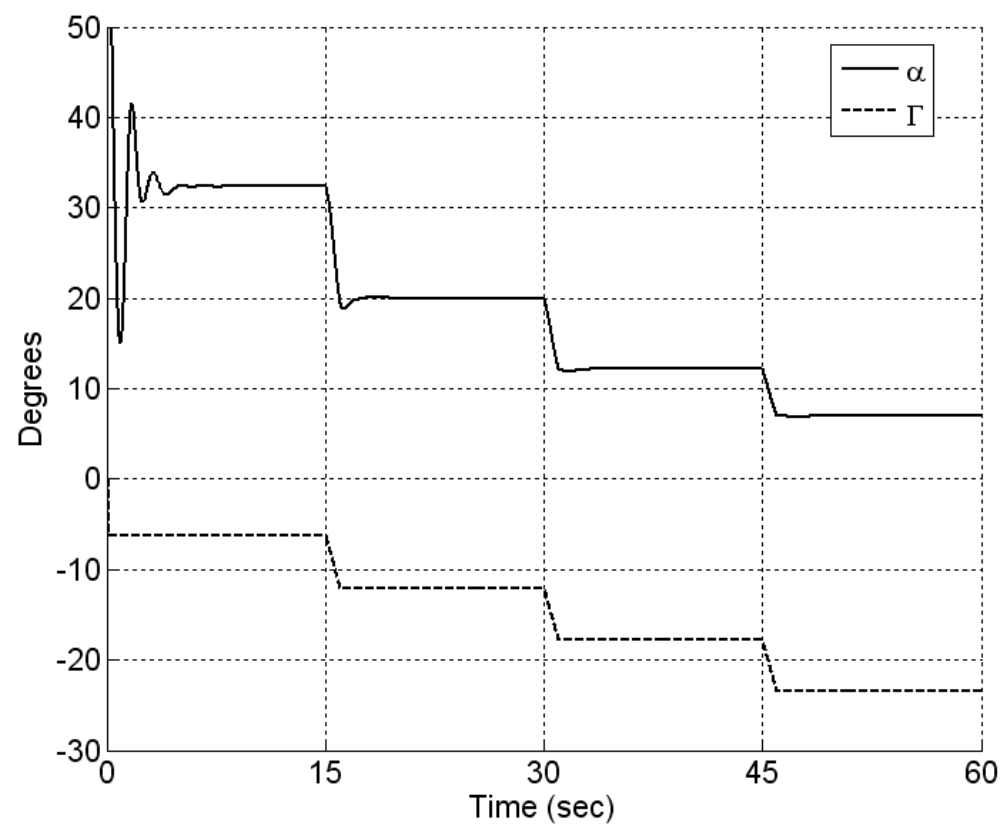

Figure 13. Simulated Angle of Attack Varying Incidence $\Gamma$ of System 1

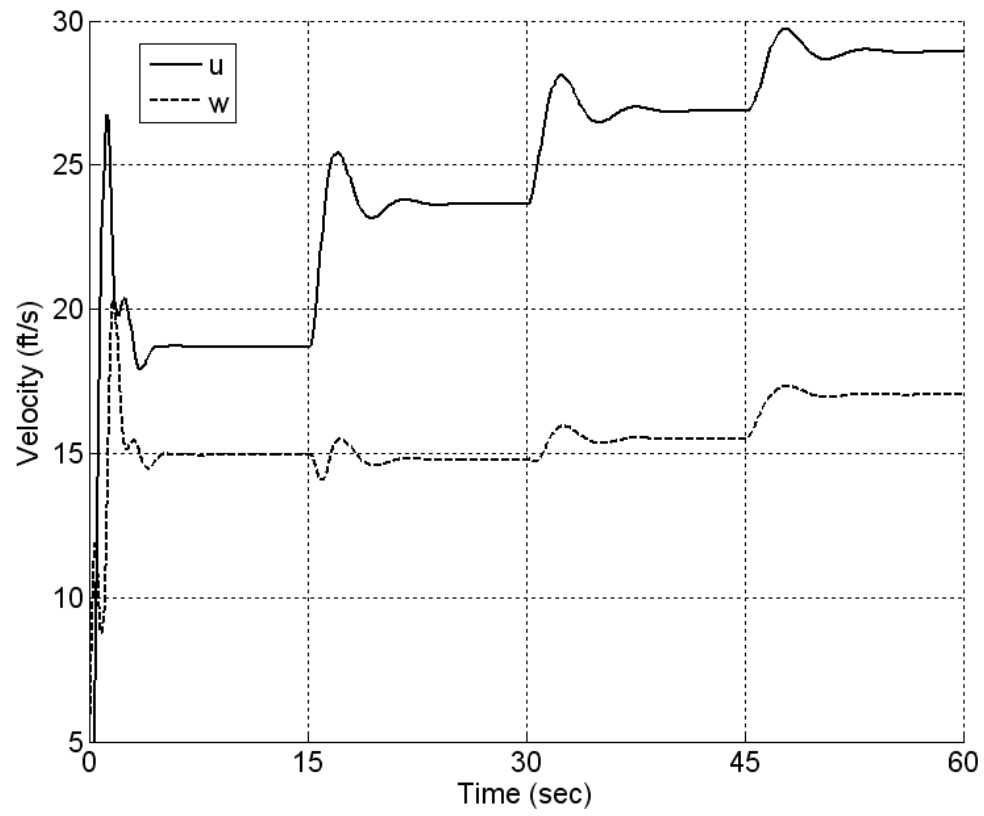

Figure 14. Simulated Velocities Varying Incidence $\Gamma$ of System 1

\section{Precision Placement Algorithm}


The precision placement algorithm is based on a Model Predictive Control (MPC) scheme that tracks desired heading and was successfully demonstrated by Slegers and Costello. ${ }^{6}$ Consider a SISO discrete system described in state space form as given in Eq. (22).

$$
\begin{aligned}
& x_{k+1}=A x_{k}+B u_{k} \\
& y_{k}=C x_{k}
\end{aligned}
$$

Assume the system matrices $A, B$ and $C$ are known and that $x_{k}$ is the state vector $\left[\begin{array}{llll}\phi_{k} & \psi_{k} & p_{k} & r_{k}\end{array}\right]^{T}, u_{k}$ is the asymmetric brake deflection $\delta_{a}$, and $y_{k}$ is the output $\psi_{k}$ at time $k$. The model described above can be used to estimate the future state of the system. Assuming a desired trajectory is known an estimated error signal $\tilde{e}_{k}=w_{k}-\tilde{y}_{k}$ is computed over a finite set of future time instants called the prediction horizon, $H_{p}$, where $w_{k}$ is the desired output and the symbol $\sim$ is used to represent an estimated quantity. In model predictive control, the control computation problem is cast as a finite time discrete optimal control problem. To compute the control input at a given time instant, a quadratic cost function is minimized through the selection of the control history over the control horizon. The cost function can be written as:

$$
J=(W-\tilde{Y})^{T}(W-\tilde{Y})+U^{T} R U
$$

where,

$$
\begin{gathered}
W=\left\{\begin{array}{llll}
w_{k+1} & w_{k+2} & \cdots & w_{k+H_{p}}
\end{array}\right\}^{T} \\
\tilde{Y}=K_{C A} x_{k}+K_{C A B} U \\
U=\left\{\begin{array}{llll}
u_{k} & u_{k} & \cdots & u_{k+H_{p}-1}
\end{array}\right\}^{T}
\end{gathered}
$$

and $R$ is a symmetric positive semi-definite matrix of size $H_{p}$. Equation 25 is used to express the predicted output vector $\tilde{Y}$ in terms of the system matrices.

$$
\begin{gathered}
K_{C A}=\left[\begin{array}{c}
C A \\
C A^{2} \\
\vdots \\
C A^{H P}
\end{array}\right] \\
K_{C A B}=\left[\begin{array}{ccccc}
C B & 0 & 0 & 0 & 0 \\
C A B & C B & 0 & 0 & 0 \\
C A^{2} B & C A B & C B & 0 & 0 \\
\vdots & \vdots & & \ddots & 0 \\
C A^{H_{p}-1} B & \cdots & C A^{2} B & C A B & C B
\end{array}\right]
\end{gathered}
$$

Equations 25 and 26 can be substituted into the cost function of Eq. (23) resulting in Eq. (29) that is in terms of the system state $x_{k}$, desired trajectory $W$, control vector $U$ and system matrices $A, B, C$, and $R$. 


$$
J=\left(W-K_{C A} x_{k}-K_{C A B} U-K_{C A D 1}\right)^{T}\left(W-K_{C A} x_{k}-K_{C A B} U-K_{C A D 1}\right)+U^{T} R U
$$

The control $U$, which minimizes Eq. (29) is

$$
U=\mathrm{K}\left(W-K_{C A} x_{k}-K_{C A D 1}\right)
$$

where,

$$
\mathrm{K}=\left(K_{C A B}^{T} K_{C A B}+R\right)^{-1} K_{C A B}^{T}
$$

Equation 30 contains the optimal control inputs over the entire control horizon, however at time $\mathrm{k}$ only the first element $u_{k}$ is needed. The first element $u_{k}$ can be extracted from Eq. (30) by defining $\mathrm{K}_{1}$ as the first row of $\mathrm{K}$. The optimal control over the next time sample becomes

$$
u_{k}=\mathrm{K}_{1}\left(W-K_{C A} x_{k}-K_{C A D 1}\right)
$$

The MPC algorithm requires a desired trajectory heading which is defined by four parameters: Target Location, Away Distance, Cycle Distance and Wind Heading Angle. As shown in Fig. 15, these four parameters define three fixed tracking points: Target Point, Away Point and Cycle Point. Using these three points, precision placement objectives are divided into four phases when glide slope control is implemented and five phases otherwise. The phases are pictured in Fig. 15 and defined as follows:

Phase1: System travels a direct path to the Cycle Point. The system is released up wind to ensure it reaches the target in strong winds.

Phase 2: System circles around the Away and Cycle Point. Down wind glide slope is estimated when traveling toward the Target Point. This continues until the Switch Altitude is reached. The Switch Altitude is defined as the distance to the target divided by the estimated glide slope plus an excess altitude. Excess altitude is only required when glide slope control is absent. Excess altitude allows the system to turn to the target early because when GS control is absent the effective GS cannot be increased only reduced by swerving.

Phase 3: System travels directly to the Away Point. Glide slope estimation is terminated.

Phase 4:

No GS Control - System continues glide slope estimation. At each update time the distance to the target is calculated and a distance to waste is calculated. MPC tracks an "S" trajectory turning left and right to waste an appropriate distance to impact the target.

GS Control - System continues glide slope estimation. At each update time the angular velocity of the vector from the system to target is calculated. A Proportional-Integral controller using incidence angle as control is implemented to track zero angular velocity, thus placing the system on the required GS to impact the target. MPC tracks a path directly to the target.

Phase 5: The system flies directly to the target once a Critical Altitude is achieved. 


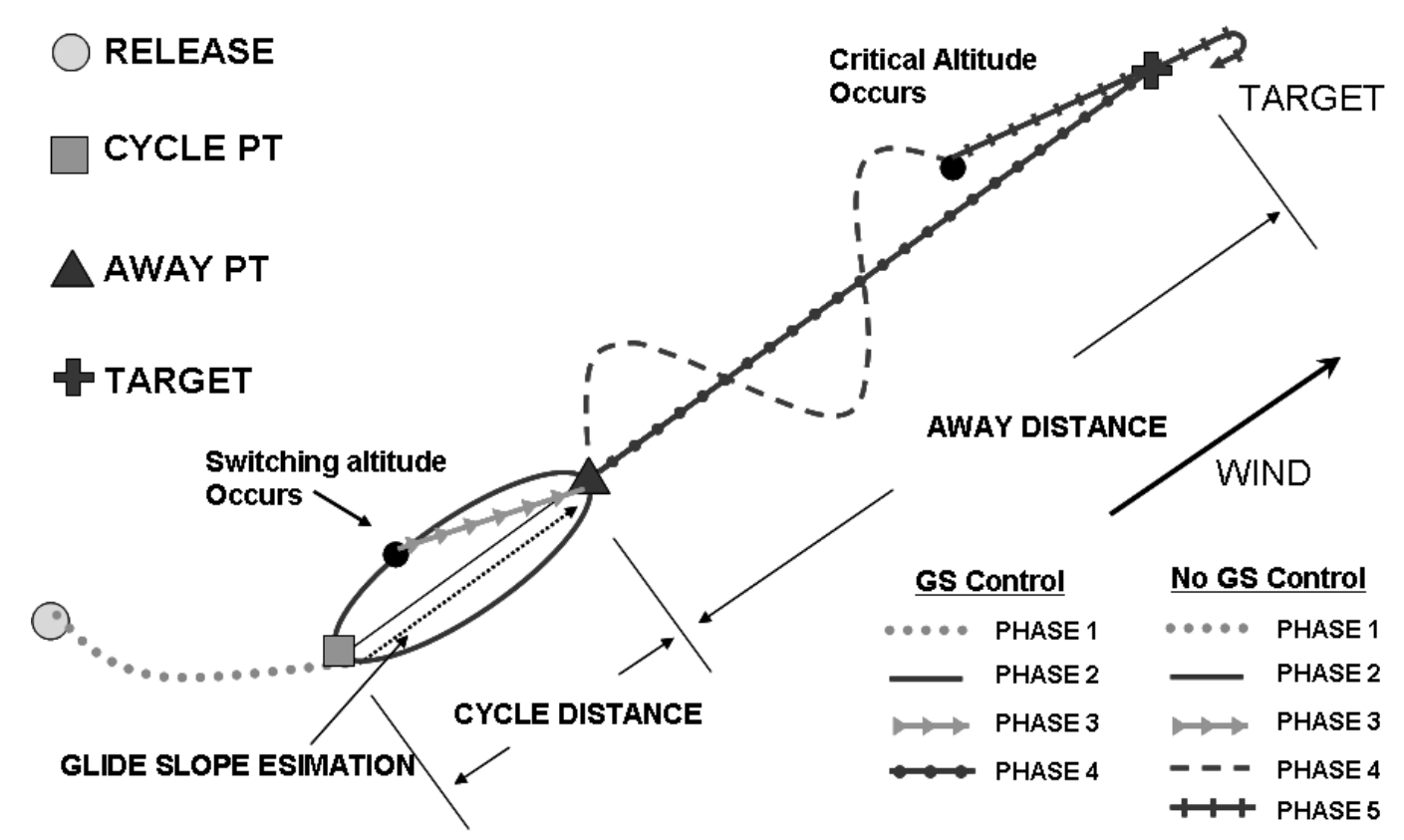

Figure 15. Phases of Precision Placement Algorithm

Nominal simulations of the precision placement algorithm with MPC were done for the canopy and payload of system 1. The nominal incidence is -12 deg and physical parameters are listed in Table 3 . The discrete linear matrices used for MPC are provided in Eqs. (33) and (34). The system is released from an altitude of $2500 \mathrm{ft}, 2500$ $\mathrm{ft}$ down range and $150 \mathrm{ft}$ cross range with a desired target at the origin. Away and Cycle Distance are 2000 and 1000 $\mathrm{ft}$ respectively. Without GS control the Critical and Excess Altitudes are 100 and $200 \mathrm{ft}$ respectively. A $5 \mathrm{ft} / \mathrm{s}$ tail wind rotated 10 deg with respect to the target line is used both with and without GS control. Results are shown in Figs. 16 through 20 where both methods impact within $15 \mathrm{ft}$ of the target. Phases 1 and 2 are identical for both methods. Phase 3 is entered sooner without GS control as seen in Figs. 16 and 17 because of the required excess altitude. Phase 3 is entered at an altitude of $1250 \mathrm{ft}$ at $103 \mathrm{sec}$ without GS control and $1050 \mathrm{ft}$ at 111 sec with GS control. Phase 4 is entered at 132 sec without GS control and at $140 \mathrm{sec}$ with GS control. During phase 4 the system with GS control varies the canopy incidence and angle of attack to adjust for errors in GS as seen in Figs. 18 and 19. The system without GS control swerves left and right to adjust the effective GS requires more active brake maneuvers in the final stages as shown in Fig. 20. An advantage of GS control is seen during the fourth phase. Over the last $2000 \mathrm{ft}$ of range the system with GS control maintains a constant orientation with respect to the target and wind enabling accurate GS estimation. Swerving required by the system without GS control changes the orientation with respect to the wind. As the system faces toward positive and negative cross range the wind slows and increases the forward speed respectively. Changing speeds make accurate GS estimation more difficult while the variation in cross range induces additional errors in impact.

Table 3. Parafoil and Payload Physical Parameters

\begin{tabular}{|c|c|c|}
\hline Variable & Value & Units \\
\hline$I_{X X}$ & 0.312 & $\operatorname{slug} \cdot f t^{2}$ \\
\hline$I_{Y Y}$ & 0.296 & $\operatorname{slug} \cdot f t^{2}$ \\
\hline$I_{Z Z}$ & 0.039 & $\operatorname{slug} \cdot f t^{2}$ \\
\hline$I_{X Z}$ & 0.022 & $\operatorname{slug} \cdot f t^{2}$ \\
\hline$r$ & -0.20 & - \\
\hline
\end{tabular}




\begin{tabular}{|c|c|c|}
\hline$C_{l p}$ & -0.15 & - \\
\hline$C_{l \delta a}$ & -0.005 & - \\
\hline$C_{m q}$ & -0.40 & - \\
\hline$C_{n r}$ & -0.09 & - \\
\hline$C_{n \delta a}$ & 0.007 & - \\
\hline$C_{D S}$ & 0.30 & $f t^{2}$ \\
\hline$S_{S}$ & 0.65 & $\operatorname{slug}$ \\
\hline$A$ & 0.0008 & $\operatorname{slug}$ \\
\hline$B$ & 0.0022 & $\operatorname{slug}$ \\
\hline$C$ & 0.0290 & $\operatorname{slug}$ \\
\hline$F$ & 0.0010 & $\operatorname{slug} \cdot f t^{2}$ \\
\hline$P$ & 0.040 & $\operatorname{slug} \cdot f t^{2}$ \\
\hline$Q$ & 0.100 & $\operatorname{slug} \cdot f t^{2}$ \\
\hline$R$ & 0.0018 & \\
\hline
\end{tabular}

$$
\begin{gathered}
A=\left[\begin{array}{cccc}
0.899 & 0 & 0.180 & 0.020 \\
0.008 & 1.000 & 0.001 & 0.033 \\
-0.119 & 0 & -0.017 & -0.002 \\
0.008 & 0 & 0.002 & 0.000
\end{array}\right] \\
B=\left[\begin{array}{c}
0.001 \\
0.101 \\
-0.012 \\
0.104
\end{array}\right]
\end{gathered}
$$




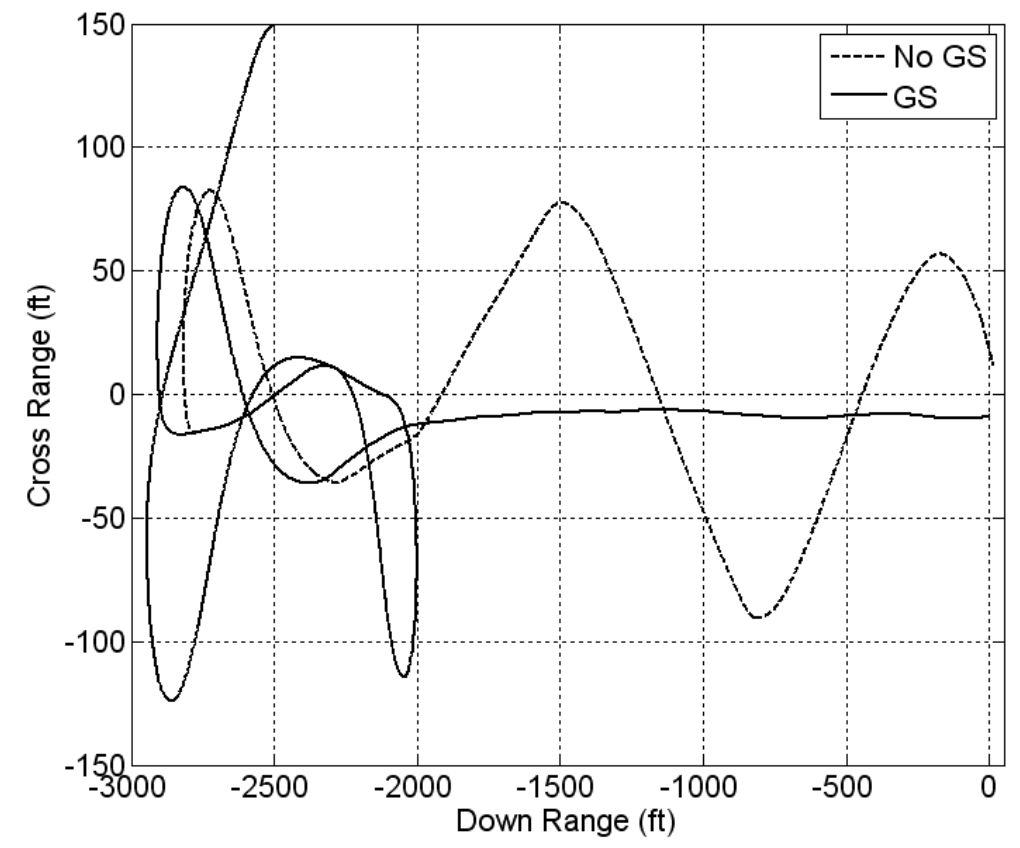

Figure 16. Simulated Precision Placement Cross Range

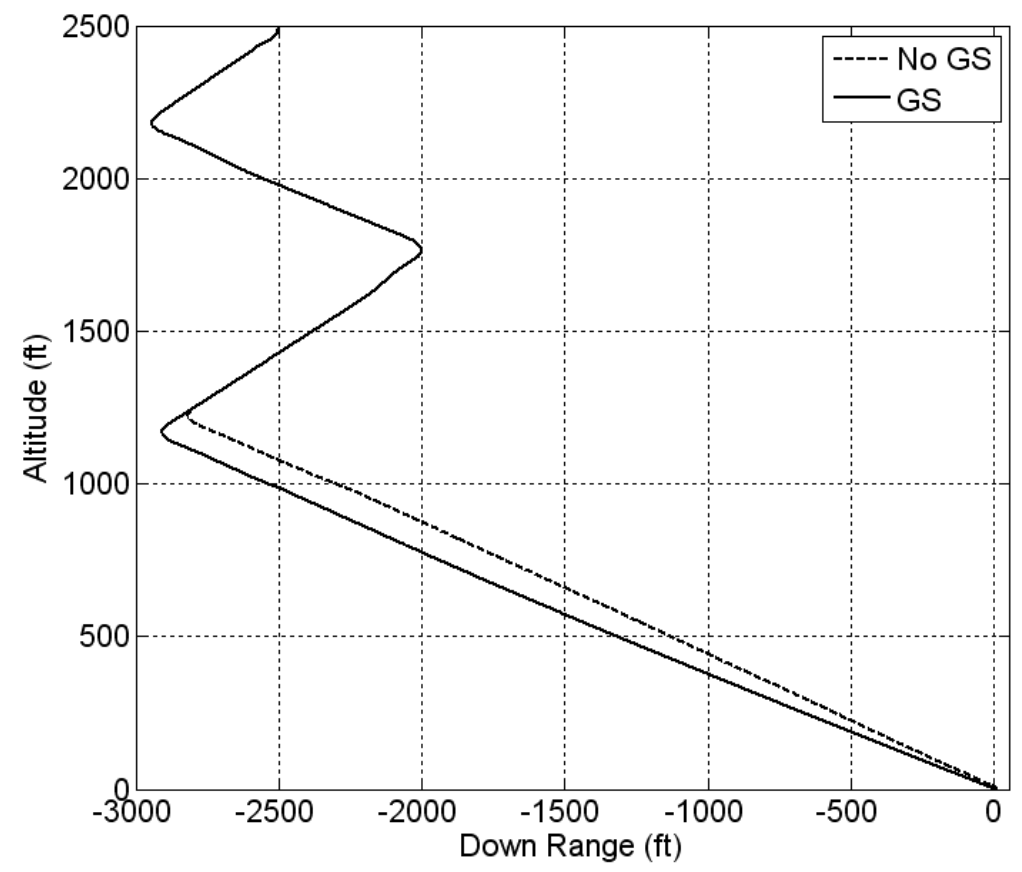

Figure 17. Simulated Precision Placement Altitude 


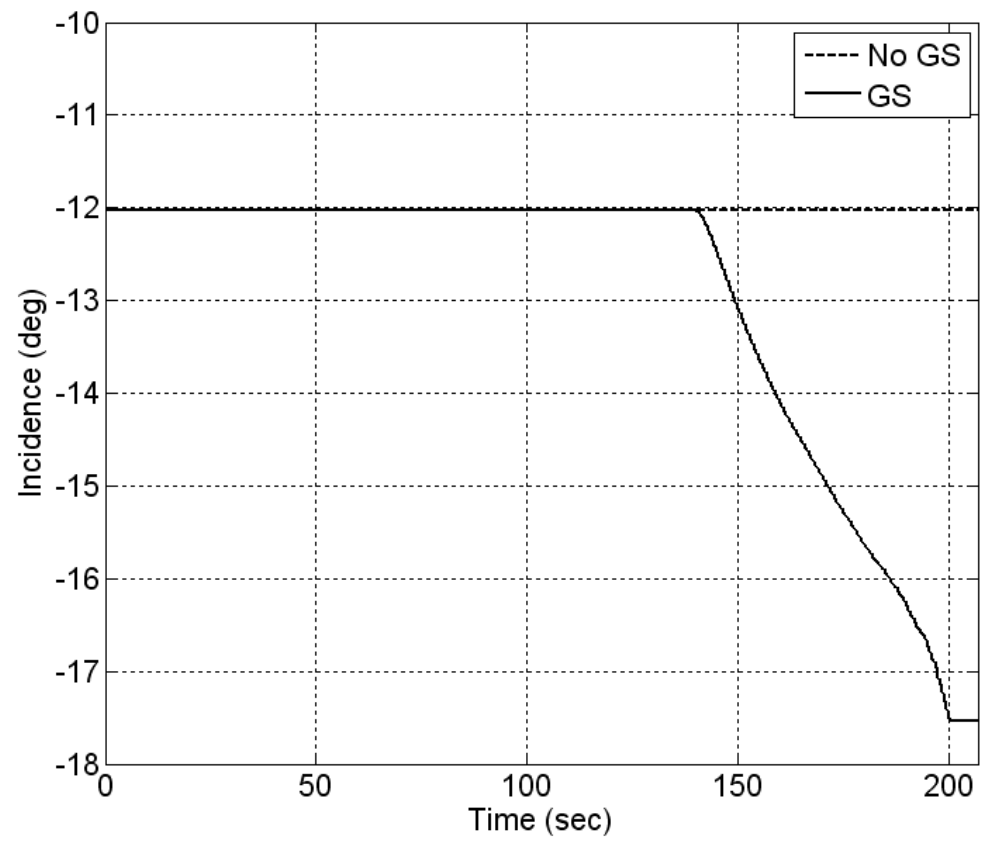

Figure 18. Simulated Precision Placement Incidence Angle

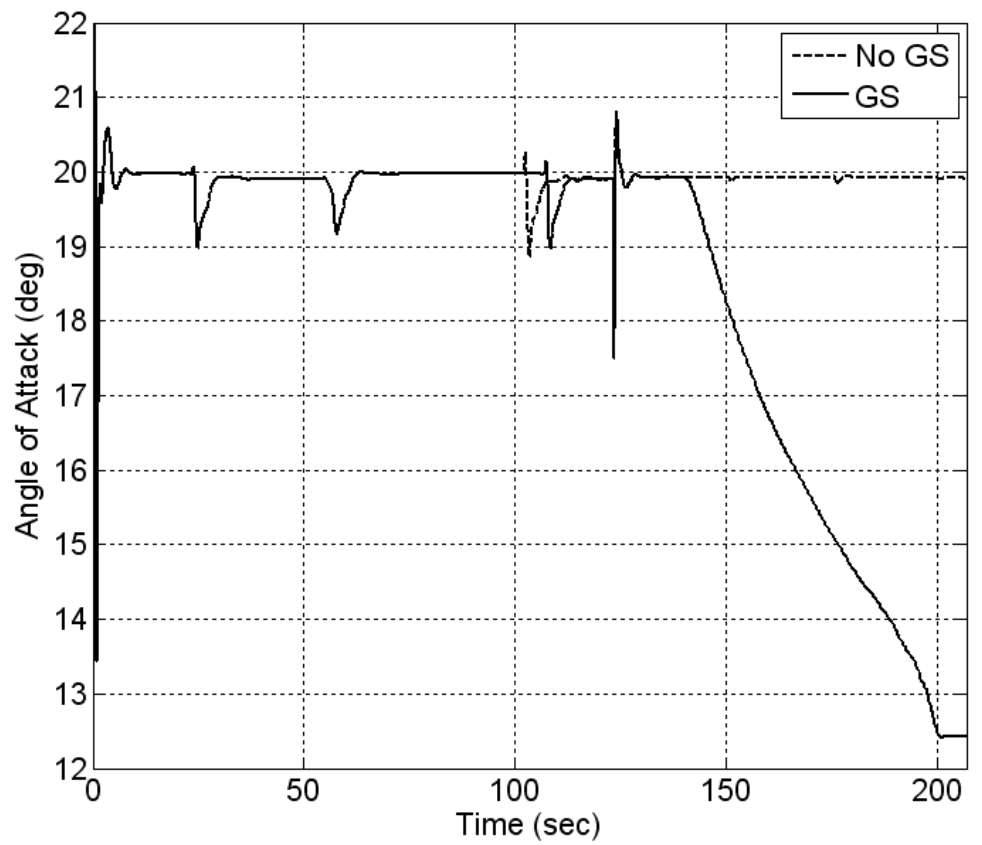

Figure 19. Simulated Precision Placement Angle of Attack 


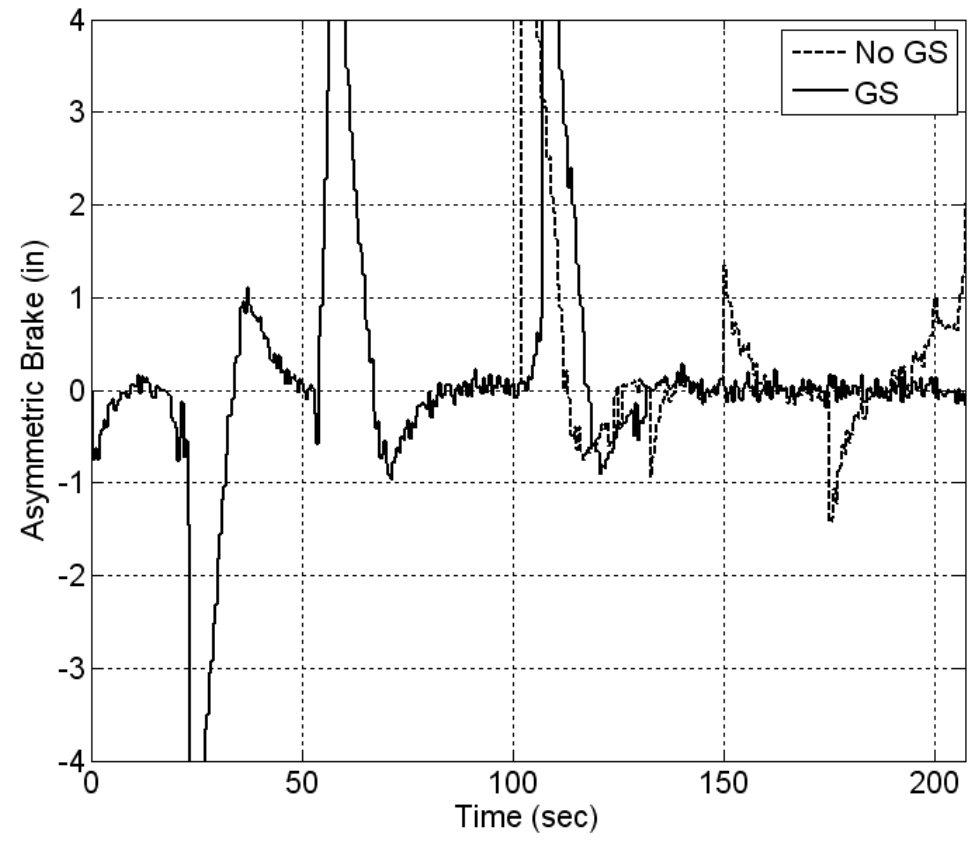

Figure 20. Simulated Precision Placement Asymmetric Control.

\section{Precision Placement Results}

Monte Carlo simulations of 100 drops were completed using the Precision Placement Algorithm with MPC. Noise was injected into GPS, Altitude, and IMU sensors. In addition to sensor errors, three sources of wind variation were added to the simulation; wind shear, varying magnitude and direction. The wind was divided into two segments varied independently, namely, wind above $1000 \mathrm{ft}$ and wind below $1000 \mathrm{ft}$ in order to simulated inconsistent wind profiles. Prevailing wind was assumed by the system to come from a heading of zero degrees while true wind varied in its direction. For all simulations, the target was set as the origin. Sensor noise and wind variation statistics are listed in Table 3.

Table 3. Error Statistics

\begin{tabular}{|c|l|c|}
\hline Parameter & Mean & Standard Deviation \\
\hline Initial Condition Position X & $3500 \mathrm{ft}$ & $750 \mathrm{ft}$ \\
\hline Initial Condition Position Y & $0 \mathrm{ft}$ & $750 \mathrm{ft}$ \\
\hline Initial Condition Position Z & $4500 \mathrm{ft}$ & $750 \mathrm{ft}$ \\
\hline GPS X Bias direction & $0.0 \mathrm{ft}$ & $3.0 \mathrm{ft}$ \\
\hline GPS Y Bias direction & $0.0 \mathrm{ft}$ & $3.0 \mathrm{ft}$ \\
\hline GPS X Deviation direction & $1.0 \mathrm{ft}$ & $0.0 \mathrm{ft}$ \\
\hline GPS Y Deviation direction & $1.0 \mathrm{ft}$ & $0.0 \mathrm{ft}$ \\
\hline Altitude Bias direction & $0.0 \mathrm{ft}$ & $5.0 \mathrm{ft}$ \\
\hline Altitude Variation direction & $1.0 \mathrm{ft}$ & $0.0 \mathrm{ft}$ \\
\hline Roll, Pitch and Yaw Bias & $0.0 \mathrm{deg}$ & $1.7 \mathrm{deg}$ \\
\hline Roll, Pitch and Yaw Deviation & $1.7 \mathrm{deg}$ & $0.0 \mathrm{deg}$ \\
\hline u,v and w Bias & $0.0 \mathrm{ft} / \mathrm{s}$ & $0.1 \mathrm{ft} / \mathrm{s}$ \\
\hline u,v and w Deviation & $0.7 \mathrm{ft} / \mathrm{s}$ & $0.0 \mathrm{ft} / \mathrm{s}$ \\
\hline p, q, and r Bias & $0.0 \mathrm{deg}$ & $1.7 \mathrm{deg}$ \\
\hline p, q, and r Deviation & $1.0 \mathrm{deg}$ & $0.0 \mathrm{deg}$ \\
\hline Wind1 & $10.0 \mathrm{ft} / \mathrm{s}$ & $3.0 \mathrm{ft} / \mathrm{s}$ \\
\hline Wind2 & $10.0 \mathrm{ft} / \mathrm{s}$ & $3.0 \mathrm{ft} / \mathrm{s}$ \\
\hline Wind Heading Error & $0.0 \mathrm{deg}$ & $11.0 \mathrm{deg}$ \\
\hline
\end{tabular}


Monte Carlo simulations were first completed with and without GS control including sensor errors and no wind. Dispersion results are shown in Fig. 21 while histograms are provided in Figs. 22 and 23. The circular error probable (CEP) defined by the radius which includes 50 percent of the impacts are shown by a circle. CEP with and without GS control are 9.8 and $13.2 \mathrm{ft}$ respectively with dispersion patterns being similar in both cases. The main difference is found in the histograms where without GS control impacts are skewed toward larger errors, where 5\% of impacts have more than $30 \mathrm{ft}$ of error. With GS control no impacts have more than $30 \mathrm{ft}$ of error.
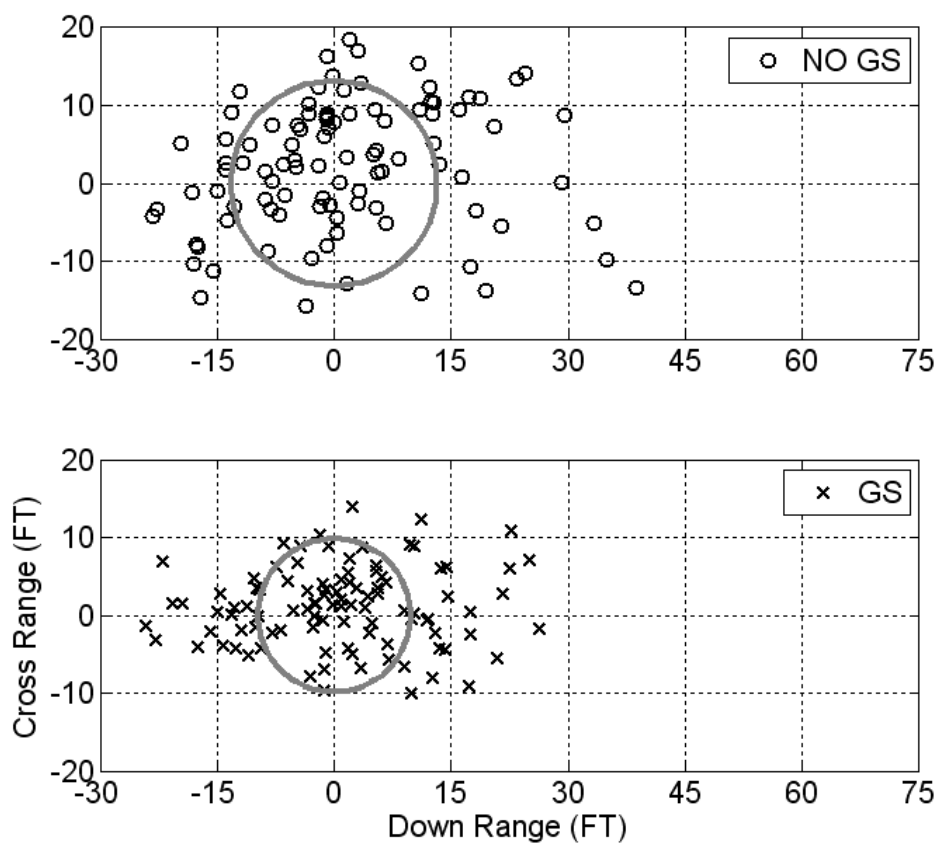

Figure 21. Dispersion for All Sensor Errors and No Wind

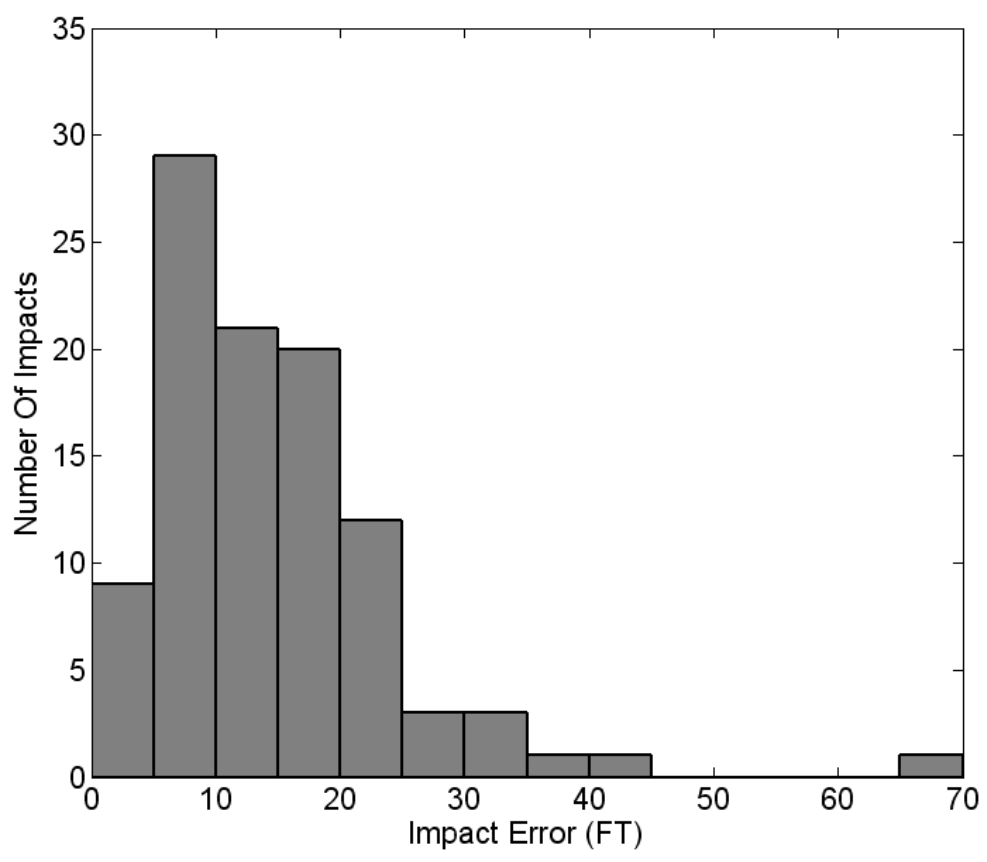


Figure 22. Without GS Control Histogram for Sensor Errors and No Wind

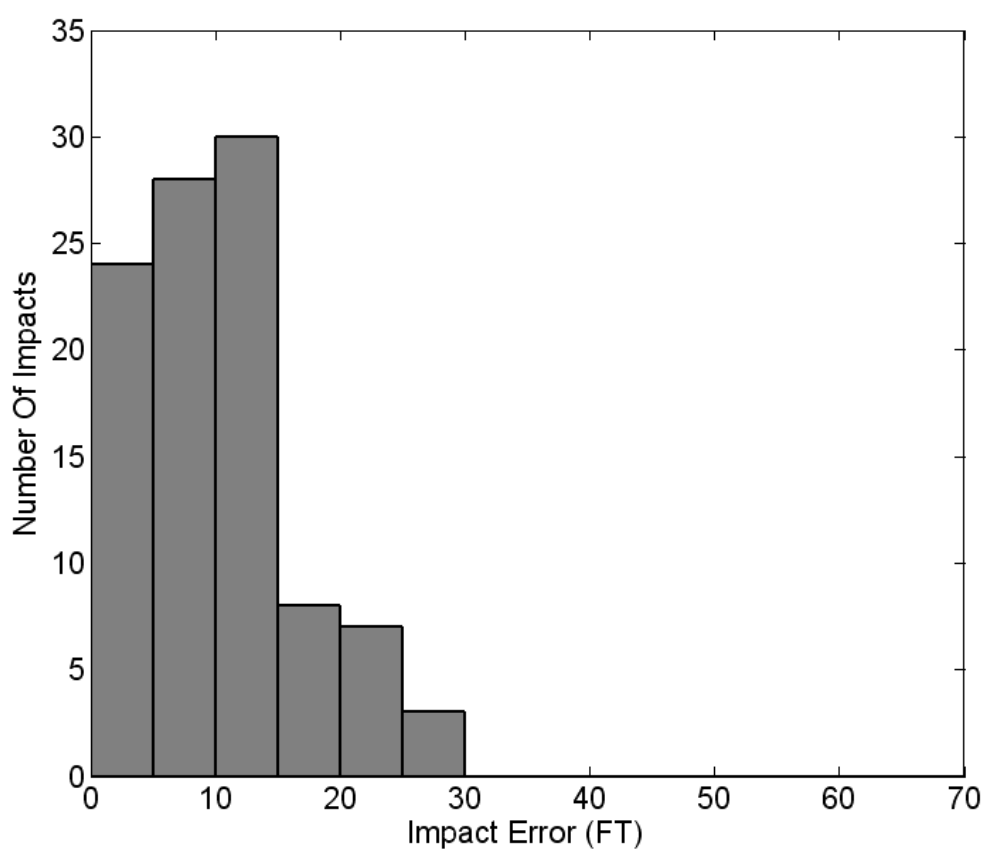

Figure 23. GS Control Histogram for Sensor Errors and No Wind

Results including both sensor errors and wind variations are shown in Figs. 24 through 26. CEP with and without GS control are 16.7 and $72.5 \mathrm{ft}$ respectively. Including winds, the GS control CEP increased by only 70 percent while the CEP without GS control increased 450 percent. Including GS control a reduction by more than a factor of three is achieved in CEP and sensitivity to winds is reduced. Dispersion patterns also differ significantly. With GS control the dispersion in mainly in range with 97 percent of the cases having less than $20 \mathrm{ft}$ of cross range error. Swerving required without GS control increased dispersion in cross range. GS control also reduced errors greater than $200 \mathrm{ft}$ from 11 to 1 . 

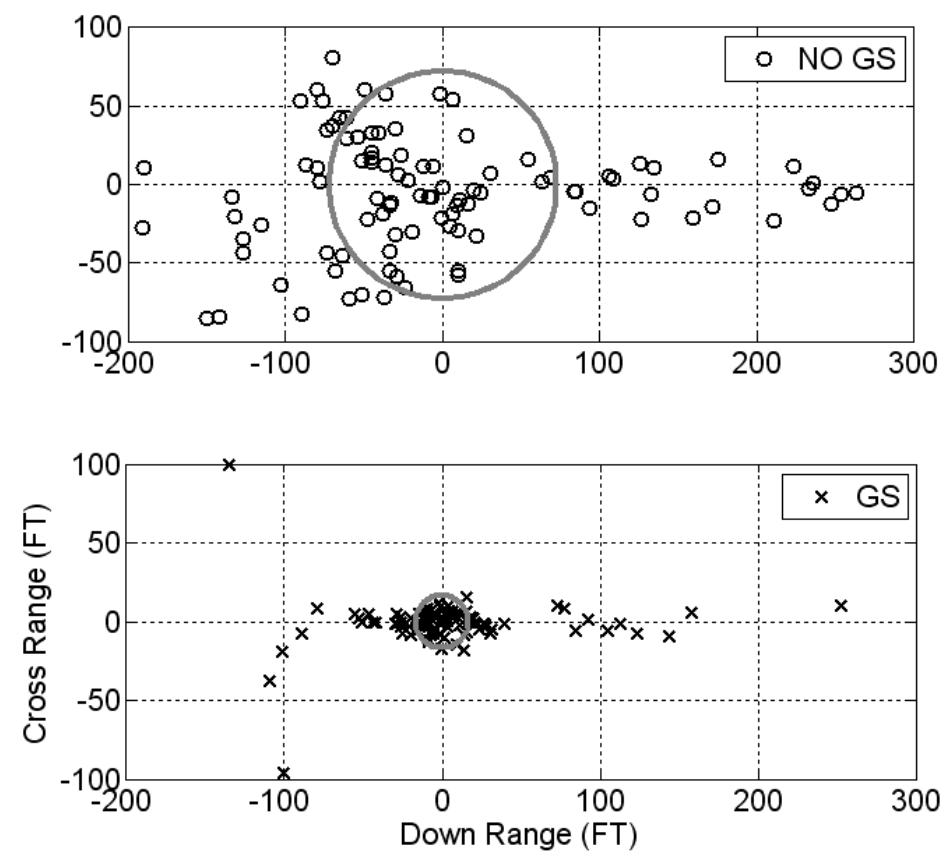

Figure 24. Dispersion for All Sensor Errors and Varying Wind

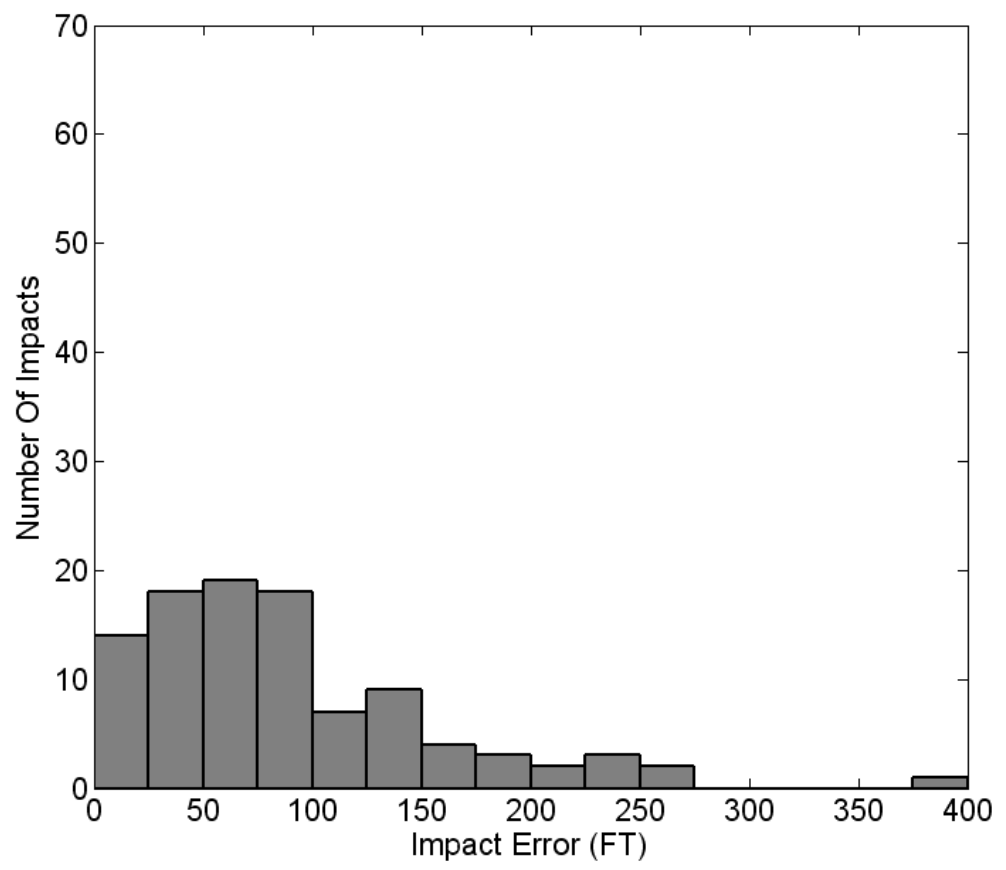

Figure 25. No GS Control Histogram for Sensor Errors and Varying Winds 


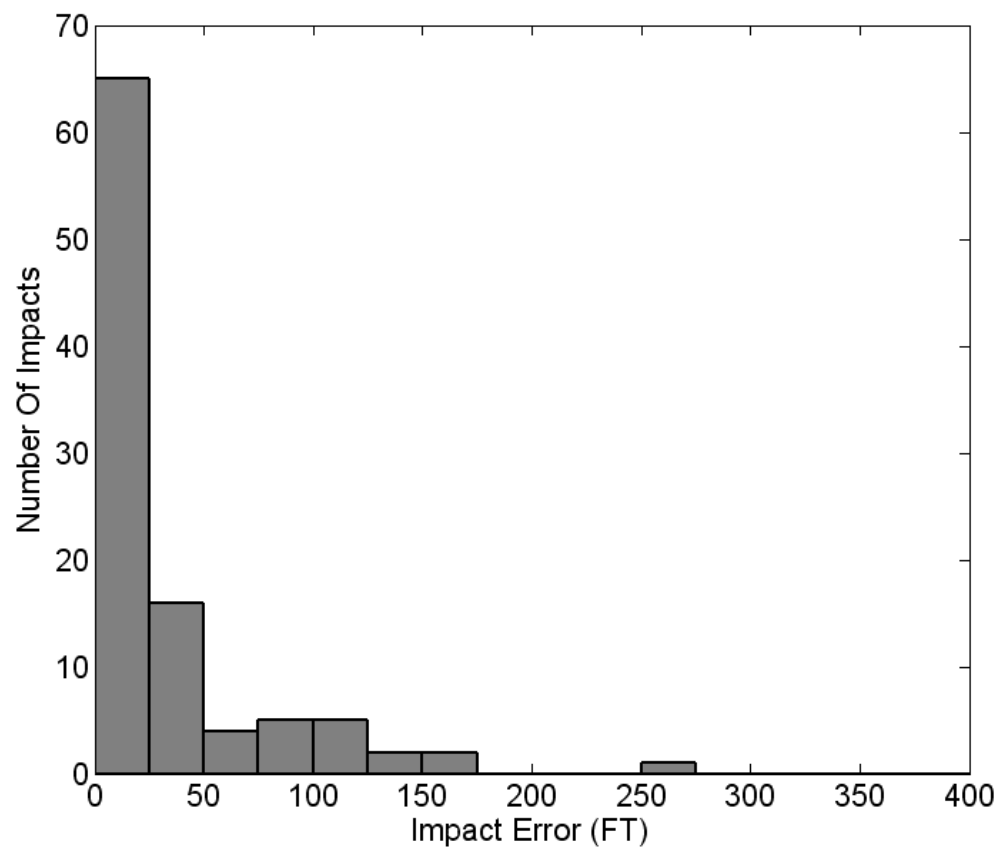

Figure 26. GS Control Histogram for Sensor Errors and Varying Winds

\section{Conclusion}

Glide slope control was demonstrated with two parafoil systems. One system experienced increased glide slope from decreasing the incidence angle wile the other experienced the opposite. The location of the system's angle of attack on the L/D curve determined the response. Parafoils of higher efficiency possessing a smaller thickness to chord ratios, larger aspect ratios and rounded nose may be able to operate to the left of their maximum L/D. Inefficient canopies may tend to operate to the right. Effective GS control can be achieved in either case. GS control becomes minimal when operating near the maximum L/D.

Precision placement was demonstrated with and without GS control. Without GS control the parafoil swerves left and right to change the effective GS towards the target. With GS control the incidence angle is controlled to place the system on a desired GS while traveling directly towards the target. Monte Carlo simulations completed with sensor errors and no wind showed successful precision placement in both cases. Using GS control the CEP was slightly lower, $9.8 \mathrm{ft}$ compared to $13.2 \mathrm{ft}$ without. In the presence of uncertain winds GS control reduced the CEP by a factor three compared to no GS control. The resulting improvement was a result of improved GS estimation by traveling the same direction and a reduction in cross range error by not requiring swerve maneuvers.

\section{References}

${ }^{1}$ N. Slegers, M. Costello, “Aspects of Control for a Parafoil and Payload System,” Journal of Guidance, Control, and Dynamics, Vol 26, No 6, pp 898-905, 2003.

${ }^{2}$ G. Ware, J. Hassell, "Wind-Tunnel Investigation of Ram-Air_Inflated All-Flexible Wings of Aspect Ratios 1.0 to 3.0," NASA TM SX-1923, 1969.

${ }^{3}$ G. Brown, "Parafoil Steady Turn Response to Control Input,” AIAA Paper 93-1241.

${ }^{4}$ M. Hailiang, Q. Zizeng, “9-DOF Simulation of Controllable Parafoil System for Gliding and Stability,” Journal of National University of Defense Technology, Vol. 16 No. 2, pp. 49-54, 1994.

${ }^{5}$ D. DeTurris, J. Ervin, S. Alptekin, "Development of an Autonomous Tactical Reconnaissance Platform," AIAA-2003-2117, 17th AIAA Aerodynamic Decelerator Systems Technology Conference and Seminar, Monterey, California, May 19-22, 2003.

${ }^{6} \mathrm{H}$. Altmann, J. Windl, "ParaLander: A Medium-weight Demonstrator for Autonomous, Range-optimized Aerial Cargo Delivery," AIAA-2005-1627, 18th AIAA Aerodynamic Decelerator Systems Technology Conference and Seminar, Munich, Germany, May 23-26, 2005. 
${ }^{7}$ T. Jann, "Advanced Features for Autonomous Parafoil Guidance, Navigation and Control," AIAA-2005-1642, 18th AIAA Aerodynamic Decelerator Systems Technology Conference and Seminar, Munich, Germany, May 23-26, 2005.

${ }^{8}$ T. Bennett, R. Fox, "Design, Development \& Flight Testing of the NASA X-38 $7500 \mathrm{ft} 2$ Parafoil Recovery System," AIAA2003-2107, 17th AIAA Aerodynamic Decelerator Systems Technology Conference and Seminar, Monterey, California, May 1922, 2003.

${ }^{9}$ C. Madsen, R. Sostaric, C. Cerimele, "Flight Performance, Aerodynamics, and Simulation Development for the X-38 Parafoil Test Program," AIAA-2003-2108, 17th AIAA Aerodynamic Decelerator Systems Technology Conference and Seminar, Monterey, California, May 19-22, 2003.

${ }^{10}$ S. George, D. Carter, J. Berland, S. Dunker, S. Tavan, J. Barber, "The Dragonfly 4,500 kg Class Guided Airdrop System," AIAA-2005-7095, Infotech@Aerospace, Arlington, Virginia, Sep. 26-29, 2005.

${ }^{11}$ Y. Zhu, M. Moreau, M. Accorsi, J. Leonard, J. Smith, “Computer Simulation of Parafoil Dynamics,” AIAA 2001-2005, AIAA $16^{\text {th }}$ Aerodynamic Decelerator Systems Technology Conference, May 2001.

${ }^{12}$ M. Gupta, Z. Xu, W. Zhang, M. Accorsi, J. Leonard, R. Benney, K. Stein., "Recent Advances in Structural Modeling of Parachute Dynamics,” AIAA 2001-2030, AIAA 16 ${ }^{\text {th }}$ Aerodynamic Decelerator Systems Technology Conference, May 2001.

${ }^{13}$ C. Iacomini, C. Cerimele, “Lateral-Directional Aerodynamics from a Large Scale Parafoil Test Program,” AIAA Paper 991731.

${ }^{14}$ C. Iacomini, C. Cerimele, “Longitudinal Aerodynamics from a Large Scale Parafoil Test Program,” AIAA Paper 99-1732.

${ }^{15}$ H. Lamb, Hydrodynamics, Dover Publications, New York, pp 160-174, 1945.

${ }^{16}$ P. Lissaman, G. Brown, “Apparent Mass Effects on Parafoil Dynamics,” AIAA Paper 93-1236, 1993.

${ }^{17}$ T. Barrows, “Apparent Mass of Parafoils with Spanwise Camber,” AIAA Paper 2001-2006, 2001. 